\title{
Total Synthesis of Cinnamodial-Based Dimer (-)-Capsicodendrin
}

Youngho Jang and Sunkyu Han*

Department of Chemistry, Korea Advanced Institute of Science and Technology (KAIST), Daejeon 34141, Korea

\section{Supporting Information}

1. Determination of Enantiomeric Excess (\% ee) of (-)-Cinnamodial (1)

2. Comparison of NMR Data of Our Synthetic Natural Products with Other Reports

2.1. (-)-Cinnamodial (1)

2.2. (-)-Capsicodendrin (3)

2.3. (-)-Cinnafragolide (5)

S08

3. Computational Details

4. Structures and Energies of Cinnamodical (1), Cinnafragrin A (2), and Capsicodendrin (3)

6. References

7. Copies of ${ }^{1} \mathrm{H}$ and ${ }^{13} \mathrm{C}$ NMR Spectra of Newly Synthesized Compounds 


\section{Determination of Enantiomeric Excess (\% ee) of (-)-Cinnamodial (1)}

\section{HPLC conditions:}

Column: Daicel Chiralcel OJ-H, $4.6 \mathrm{~mm}$ x $250 \mathrm{~mm}$

Flow rate: $0.8 \mathrm{~mL} / \mathrm{min}$

Eluent: $90 \%$ hexanes, $10 \% i \mathrm{PrOH}$

Injection volume: $10 \mu \mathrm{L}$

Monitored at $\lambda: 210.8 \mathrm{~nm}$

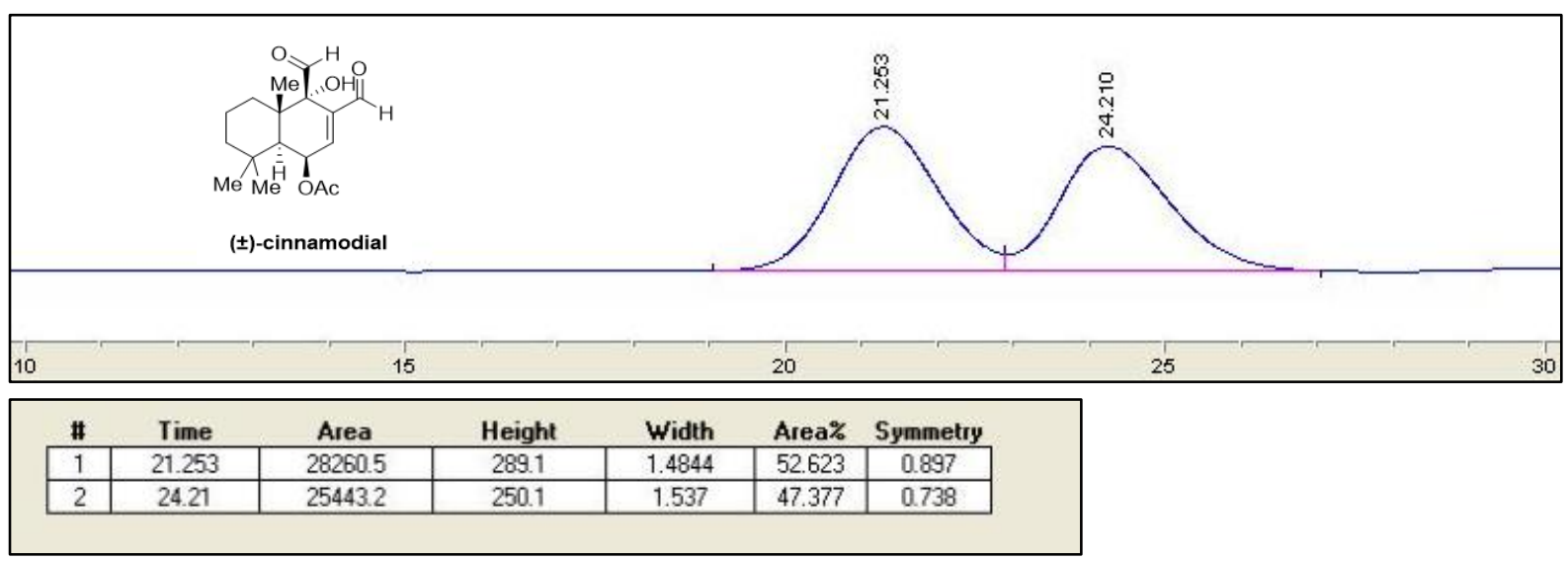

Figure S1: Chromatogram and the report of $( \pm)$-cinnamodial (1) on OJ-H column.

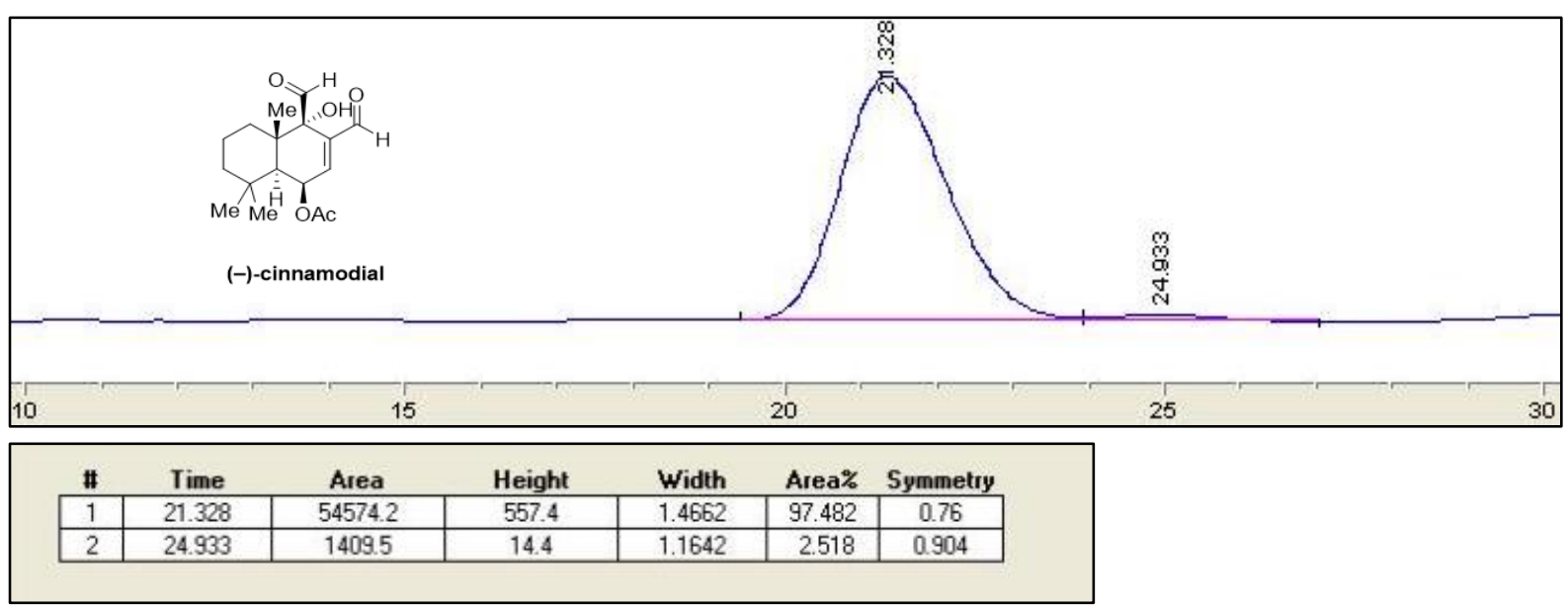

Figure S2: Chromatogram and the report of (-)-cinnamodial (1) on OJ-H column. 


\section{Comparison of NMR Data of Our Synthetic Natural Products with Other Reports}

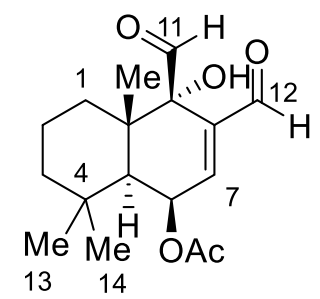

(-)-cinnamodial (1)

Table S1. Comparison of ${ }^{1} \mathrm{H}$ NMR Spectroscopic Data of (-)-Cinnamodial (1)

\begin{tabular}{cccc}
\hline position & $\begin{array}{c}\text { White group }^{1} \\
(\mathrm{ppm} ; \text { multi, } J \text { in } \\
\mathrm{Hz})\end{array}$ & $\begin{array}{c}\text { Lopez group }^{2} \\
(\mathrm{ppm} ; \text { multi, } J \text { in } \\
\mathrm{Hz})\end{array}$ & $\begin{array}{c}\text { Our group (ppm; } \\
\text { multi, } J \text { in Hz })^{34}\end{array}$ \\
\hline 5 & $2.09, \mathrm{~d}(5.0)$ & - & $2.01, \mathrm{~d}(4.8)$ \\
6 & $5.91, \mathrm{t}(5.0)$ & $5.92, \mathrm{dd}(4.0,5.0)$ & $5.86, \mathrm{t}(4.8)$ \\
7 & $6.98, \mathrm{~d}(5.0)$ & $7.02, \mathrm{~d}(5.0)$ & $6.97, \mathrm{~d}(4.7)$ \\
$9-\mathrm{OH}$ & $4.10, \mathrm{~b} \mathrm{~s}$ & $4.10, \mathrm{~b} \mathrm{~s}(1.0)$ & $4.06, \mathrm{~d}(1.3)$ \\
11 & $9.75, \mathrm{~s}$ & $9.78, \mathrm{~s}$ & $9.72, \mathrm{~d}(1.2)$ \\
12 & $9.46, \mathrm{~s}$ & $9.50, \mathrm{~s}$ & $9.44, \mathrm{~s}$ \\
$\mathrm{COMe}$ & $2.18, \mathrm{~s}$ & $2.14, \mathrm{~s}$ & $2.10, \mathrm{~s}$ \\
13 & $1.38, \mathrm{~s}$ & $1.34, \mathrm{~s}$ & $1.30, \mathrm{~s}$ \\
14 & $1.20, \mathrm{~s}$ & $1.17, \mathrm{~s}$ & $1.13, \mathrm{~s}$ \\
15 & $1.06, \mathrm{~s}$ & $1.02, \mathrm{~s}$ & $0.99, \mathrm{~s}$
\end{tabular}


Table S2. Comparison of ${ }^{13}$ C NMR Spectroscopic Data of (-)-Cinnamodial (1)

\begin{tabular}{|c|c|c|c|}
\hline position & $\begin{array}{l}\text { Harinantenaina } \\
\text { group }(\mathrm{ppm})^{5}\end{array}$ & $\begin{array}{l}\text { Mahmoud group } \\
\qquad(\mathrm{ppm})^{6}\end{array}$ & Our group $(\mathrm{ppm})^{3}$ \\
\hline $1 \mathrm{a}, \mathrm{b}$ & 44.0 & 44.2 & 44.2 \\
\hline $2 a, b$ & 32.6 & 32.6 & 32.8 \\
\hline $3 a, b$ & 17.6 & 17.7 & 17.9 \\
\hline 4 & 34.0 & 34.0 & 34.2 \\
\hline 5 & 45.0 & 45.2 & 45.1 \\
\hline 6 & 66.0 & 66.2 & 66.2 \\
\hline 7 & 148.6 & 148.5 & 148.9 \\
\hline 8 & 140.1 & 141.3 & 141.1 \\
\hline 9 & 90.0 & 77.5 & 77.5 \\
\hline 10 & 41.6 & 41.7 & 41.8 \\
\hline 11 & 193.0 & 192.9 & 193.2 \\
\hline 12 & 201.1 & 201.0 & 201.3 \\
\hline 13 & 20.0 & 19.9 & 20.1 \\
\hline 14 & 24.7 & 24.7 & 24.9 \\
\hline 15 & 31.8 & 31.9 & 32.0 \\
\hline $\mathrm{CH}_{3} \mathrm{C}=\mathrm{O}$ & 21.4 & 21.3 & 21.7 \\
\hline $\mathrm{CH}_{3} \mathrm{C}=\mathrm{O}$ & 170.0 & 170.0 & 170.3 \\
\hline
\end{tabular}


Table S3. Comparison of ${ }^{13}$ C NMR Spectroscopic Data of (-)-Capsicodendrin (3)

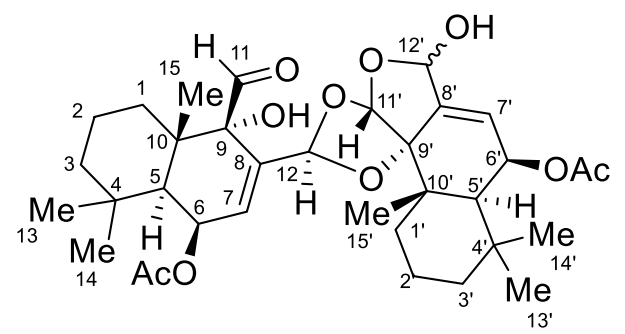

(-)-capsicodendrin (3)

\begin{tabular}{|c|c|c|c|}
\hline position & $\begin{array}{l}\text { Harinantenaina } \\
\text { group }(\mathrm{ppm})^{5}\end{array}$ & $\begin{array}{l}\text { Mahmoud group } \\
\text { (ppm)오류! 책갈피가 } \\
\text { 정의되어 있지 않습니다. }\end{array}$ & Our group $(\mathrm{ppm})^{37}$ \\
\hline
\end{tabular}

\begin{tabular}{|c|c|c|c|}
\hline \multicolumn{4}{|c|}{ Unit A } \\
\hline 1 & 44.0 & 44.2 & $44.3,44.2$ \\
\hline 2 & $32.7,32.7$ & 32.8 & $33.0,32.9$ \\
\hline 3 & 18.0 & 18.1 & 18.2 \\
\hline 4 & $33.6,33.6$ & $33.8,33.9$ & $34.1,33.9$ \\
\hline 5 & $46.3,46.0$ & $46.5,46.3$ & $46.5,46.3$ \\
\hline 6 & $66.8,66.6$ & $67.1,66.9$ & $67.1,66.9$ \\
\hline 7 & $125.0,124.7$ & $124.9,124.6$ & $125.3,125.0$ \\
\hline 8 & $140.1,138.5$ & $140.3,139.0$ & $140.4,138.8$ \\
\hline 9 & $90.4,90.0$ & $90.6,90.5$ & $90.7,90.6$ \\
\hline 10 & $41.8,41.8$ & 41.9 & $42.0,42.0$ \\
\hline 11 & $203.5,203.4$ & $203.5,203.4$ & $203.7,203.7$ \\
\hline 12 & $104.7,100.2$ & $104.8,100.6$ & $105.0,100.5$ \\
\hline 13 & $20.0,20.0$ & 20.0 & $20.3,20.3$ \\
\hline 14 & $24.6,24.6$ & $24.7,24.4$ & $24.9,24.6$ \\
\hline 15 & $32.2,32.1$ & 32.2 & $32.4,32.1$ \\
\hline
\end{tabular}




\begin{tabular}{|c|c|c|c|}
\hline $\mathrm{CH}_{3} \mathrm{C}=\mathrm{O}$ & $21.6,21.6$ & 21.6 & $21.9,21.8$ \\
\hline $\mathrm{CH}_{3} \mathrm{C}=\mathrm{O}$ & $170.0,170.0$ & $170.0,170.0$ & $170.3,170.3$ \\
\hline \multicolumn{4}{|l|}{ Unit B } \\
\hline $1^{\prime}$ & $44.6,44.6$ & $44.8,44.6$ & $44.9,44.9$ \\
\hline $2^{\prime}$ & $31.8,31.6$ & $32.2,31.9$ & $32.4,31.9$ \\
\hline $3^{\prime}$ & $17.8,17.8$ & 17.9 & $18.1,18.1$ \\
\hline $4^{\prime}$ & $33.8,33.8$ & $33.9,33.8$ & $34.1,33.9$ \\
\hline $5^{\prime}$ & $44.4,44.4$ & $44.4,44.4$ & $44.7,44.6$ \\
\hline $6^{\prime}$ & $66.1,66.0$ & $66.4,66.3$ & $66.4,66.3$ \\
\hline $7^{\prime}$ & $128.4,128.0$ & $128.5,128.0$ & $128.7,128.3$ \\
\hline $8^{\prime}$ & $134.7,133.8$ & $135.1,134.3$ & $134.9,134.1$ \\
\hline $9^{\prime}$ & $80.3,80.3$ & 80.5 & 80.6 \\
\hline $10^{\prime}$ & $36.8,36.7$ & 37.0 & $37.1,37.0$ \\
\hline $11^{\prime}$ & $102.2,100.0$ & $102.3,100.3$ & $102.5,100.3$ \\
\hline $12^{\prime}$ & $98.5,97.8$ & $98.6,98.0$ & $98.8,98.1$ \\
\hline $13^{\prime}$ & $19.6,19.6$ & 19.6 & $19.9,19.9$ \\
\hline $14^{\prime}$ & $24.3,24.3$ & $24.7,24.4$ & $24.9,24.6$ \\
\hline $15^{\prime}$ & $32.7,32.7$ & 32.6 & $32.9,32.9$ \\
\hline $\mathrm{CH}_{3} \mathrm{C}=\mathrm{O}$ & $21.5,21.4$ & 21.4 & $21.7,21.7$ \\
\hline $\mathrm{CH}_{3} \mathrm{C}=\mathrm{O}$ & $170.0,170.0$ & $170.0,170.0$ & $170.3,170.2$ \\
\hline
\end{tabular}


Figure S3. Comparison of ${ }^{1} \mathrm{H}$ NMR Spectra of Our Synthetic and Natural (-)Capsicodendrin (3) in DMSO-d

a. Harinantenaina Group ${ }^{1} \mathrm{H}$ NMR (NMR is taken after $1 \mathrm{hr}$ since its sample preparation) ${ }^{8}$

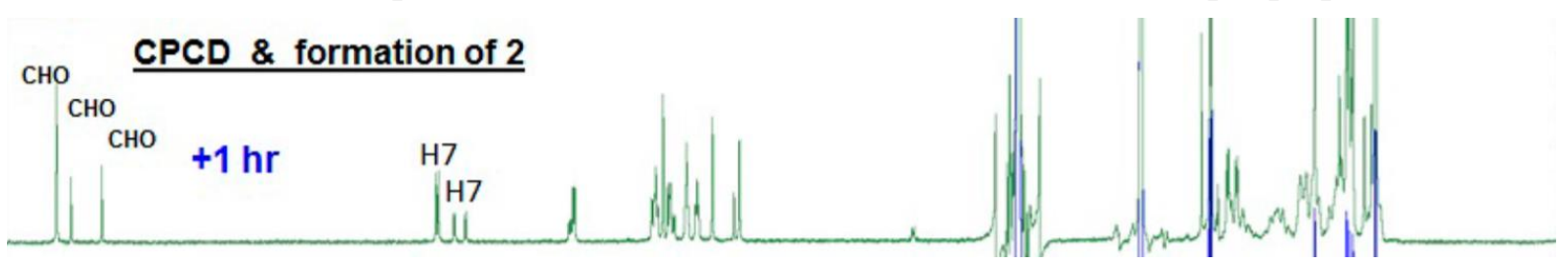

b. Our Group's ${ }^{1} \mathrm{H}$ NMR (NMR is taken after 20 min since its sample preparation)

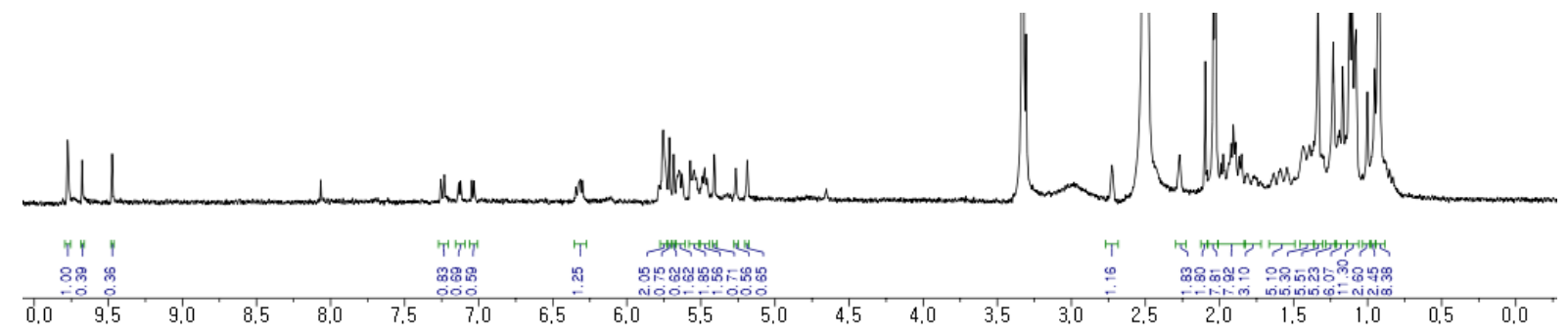

When our synthetic capsicodendrin (3) was dissolved in DMSO- $\mathrm{d}_{6}$, it underwent a rapid conversion to cinnamodial (1) consistent with Harinantenaina group's report. 
Table S4. Comparison of ${ }^{1} \mathrm{H}$ NMR Spectroscopic Data of (-)-Cinnafragolide (5)

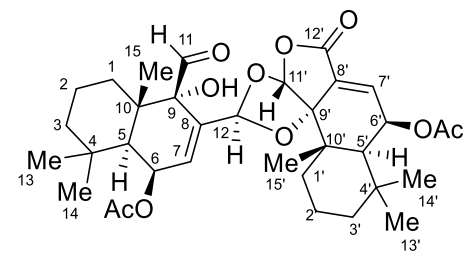

(-)-cinnafragolide (5)

\begin{tabular}{ccc}
\hline position & $\begin{array}{c}\text { Harinantenaina } \\
\text { group }(\mathrm{ppm} ; \\
\text { multi, } J \text { in } \mathrm{Hz})^{5}\end{array}$ & $\begin{array}{c}\text { Our group }(\mathrm{ppm} ; \\
\text { multi, } J \text { in } \mathrm{Hz})^{34}\end{array}$ \\
\hline Unit A & & \\
5 & $2.05, \mathrm{~d}(4.6)$ & $1.96, \mathrm{~d}(5.5)$ \\
6 & $5.82, \mathrm{dd}(4.6,3.9)$ & $5.82, \mathrm{dd}(5.4,3.4)$ \\
7 & $6.85, \mathrm{dd}(4.0,1.6)$ & $6.82, \mathrm{~d}(3.6)$ \\
11 & $9.82, \mathrm{~s}$ & $9.82, \mathrm{~d}(1.2)$ \\
12 & $5.90, \mathrm{~s}$ & $5.89, \mathrm{~s}$ \\
13 & $1.14, \mathrm{~s}$ & $1.13, \mathrm{~s}$ \\
14 & $1.12, \mathrm{~s}$ & $1.11, \mathrm{~s}$ \\
15 & $1.00, \mathrm{~s}$ & $0.98, \mathrm{~s}$ \\
$\mathrm{CH}{ }_{3} \mathrm{C}=\mathrm{O}$ & $2.08, \mathrm{~s}$ & $2.08, \mathrm{~s}$ \\
& $3.90, \mathrm{~s}$ & $3.91, \mathrm{~d}(1.4)$
\end{tabular}

Unit B

$\begin{array}{ccc}5^{\prime} & 1.89, \mathrm{~d}(4.8) & 1.93, \mathrm{~d}(4.4) \\ 6^{\prime} & 5.70, \mathrm{brt}(4.0) & 5.66, \mathrm{t}(4.5) \\ 7^{\prime} & 6.53, \text { br d (4.8) } & 6.53, \mathrm{dd}(5.2,0.9) \\ 11^{\prime} & 5.20, \mathrm{~s} & 5.39, \mathrm{~s} \\ 13^{\prime} & 1.07, \mathrm{~s} & 1.06, \mathrm{~s} \\ 14^{\prime} & 1.01, \mathrm{~s} & 1.00, \mathrm{~s}\end{array}$




$\begin{array}{ccc}15^{\prime} & 1.42, \mathrm{~s} & 1.42, \mathrm{~s} \\ \mathrm{CH}_{3} \mathrm{C}=\mathrm{O} & 2.03, \mathrm{~s} & 2.06, \mathrm{~s}\end{array}$


Table S5. Comparison of ${ }^{13}$ C NMR Spectroscopic Data of (-)-Cinnafragolide (5)

\begin{tabular}{|c|c|c|}
\hline position & $\begin{array}{l}\text { Harinantenaina } \\
\text { group }(\mathrm{ppm})^{5}\end{array}$ & Our group $(\mathrm{ppm})^{37}$ \\
\hline \multicolumn{3}{|l|}{ Unit A } \\
\hline 1 & 44.0 & 44.2 \\
\hline 2 & 32.6 & 32.9 \\
\hline 3 & 18.0 & 18.0 \\
\hline 4 & 33.8 & 34.1 \\
\hline 5 & 46.3 & 46.6 \\
\hline 6 & 66.2 & 66.4 \\
\hline 7 & 129.4 & 129.7 \\
\hline 8 & 138.5 & 138.8 \\
\hline 9 & 88.5 & 88.8 \\
\hline 10 & 41.9 & 42.2 \\
\hline 11 & 202.9 & 203.1 \\
\hline 12 & 100.1 & 100.3 \\
\hline 13 & 20.1 & 20.4 \\
\hline 14 & 24.6 & 24.9 \\
\hline 15 & 32.1 & 32.4 \\
\hline $\mathrm{CH}_{3} \mathrm{C}=\mathrm{O}$ & 170.0 & 170.2 \\
\hline $\mathrm{CH}_{3} \mathrm{C}=\mathrm{O}$ & 21.4 & 21.7 \\
\hline \multicolumn{3}{|l|}{ Unit B } \\
\hline $1^{\prime}$ & 44.4 & 44.6 \\
\hline $2^{\prime}$ & 31.0 & 31.3 \\
\hline
\end{tabular}




\begin{tabular}{|c|c|c|}
\hline $3^{\prime}$ & 17.7 & 18.0 \\
\hline $4^{\prime}$ & 33.7 & 34.0 \\
\hline $5^{\prime}$ & 44.4 & 44.7 \\
\hline $6^{\prime}$ & 65.9 & 66.2 \\
\hline $7^{\prime}$ & 127.2 & 127.5 \\
\hline $8^{\prime}$ & 132.8 & 133.0 \\
\hline $9^{\prime}$ & 80.3 & 80.6 \\
\hline $10^{\prime}$ & 37.1 & 37.4 \\
\hline $11^{\prime}$ & 101.2 & 101.4 \\
\hline $12^{\prime}$ & 166.4 & 166.6 \\
\hline $13^{\prime}$ & 19.7 & 20.0 \\
\hline $14^{\prime}$ & 24.3 & 24.5 \\
\hline $15^{\prime}$ & 32.6 & 32.9 \\
\hline $\mathrm{CH}_{3} \mathrm{C}=\mathrm{O}$ & 169.6 & 169.8 \\
\hline $\mathrm{CH}_{3} \mathrm{C}=\mathrm{O}$ & 21.3 & 21.6 \\
\hline
\end{tabular}




\section{Computational Details}

All calculations were carried out using density functional theory (DFT) ${ }^{9}$ calculations implemented in Jaguar 9.1 programs. ${ }^{10}$ For every calculation, B3LYP11 $12 \quad 13 \quad 14 \quad 15$ level of theory with Grimme's D3 dispersion correction ${ }^{16}$ was used. Geometry optimizations of all intermediates and transition states were conducted utilizing 6-31G** basis set. Single-point energies of optimized structures were obtained using Dunning's cc-pVTZ(-f) basis set ${ }^{17}$ which includes a double set of polarization functions to gas-phase optimized structure. Vibrational frequency calculations of gas-phase structures were performed using $6-31 G^{* *}$ basis set. Standard approximation was used to obtain zero-point vibrational energies and entropy corrections. Solvation energies of gas-phase optimized structures were calculated applying self-consistent reaction field (SCRF) approach ${ }^{18} 19{ }^{20}$ to solve Poisson-Boltzmann equation with dielectric constant $\varepsilon=78.4\left(\mathrm{H}_{2} \mathrm{O}\right)$. As is the case for all continuum models, the solvation energies are subjected to empirical parametrization of the atomic radii that are used to generate the solute surface. The standard set of optimized radii in Jaguar was used for H (1.150 $\AA$ ), C $(1.900 \AA)$, and $\mathrm{O}(1.600 \AA) .{ }^{21}$ The Gibbs free energies in solution phase G(sol) were obtained as follows:

$$
\begin{aligned}
& \mathrm{G}(\text { sol })=\mathrm{G}(\text { gas })+\mathrm{G}(\text { solv }) \\
& \mathrm{G}(\text { gas })=\mathrm{H}(\text { gas })-\mathrm{TS}(\text { gas }) \\
& \mathrm{H}(\text { gas })=\mathrm{E}(\mathrm{SCF})+\mathrm{ZPE} \\
& \Delta \mathrm{G}(\text { sol })=\Sigma \mathrm{G}(\text { sol }) \text { for products }-\Sigma \mathrm{G}(\text { sol }) \text { for reactants }
\end{aligned}
$$

Where $\mathrm{G}($ gas) is Gibbs free energy in gas-phase, $\mathrm{G}(\mathrm{solv})$ is solvation free energy, $\mathrm{H}$ (gas) is gasphase enthalpy, $\mathrm{S}$ (gas) is gas-phase entropy, $\mathrm{T}$ is temperature $(298.15 \mathrm{~K}) . \mathrm{E}(\mathrm{SCF})$ is selfconsistent field energy which is "raw" electronic energy, ZPE is vibrational zero-point energy. The entropy term we utilized is vibrational / rotational / translational entropy of solute(s), and the entropy of solvent is implicitly included in continuum solvation model. 


\section{Structures and Energies of Cinnamodical (1), Cinnafragrin A (2), and Capsicodendrin} (3)

DFT calculation of cinnamodial (1), and two closely related hemiacetal dimers cinnafragrin A (2) and capsicodendrin (3) was carried out to compare their solution-phase free energy. Structure of capsicodendrin (3) was adopted from the crystal structure of cinnafragrolide $^{22}$, the $\mathrm{C}-12$ ' lactone derivative of capsicodendrin. Two conformers bearing lowest solution-phase free energy of cinnafragrin A (2) were shown. Relative energies compared to two molecules of cinnamodial (1) were illustrated in $\mathrm{kcal} / \mathrm{mol}$. The energy difference between cinnafragrin A (2) conformer 1 and (12'S)-capsicodendrin (3a) is 3.72 $\mathrm{kcal} / \mathrm{mol}$. The energy difference between cinnafragrin A (2) conformer 2 and $\left(12^{\prime} R\right)-$ capsicodendrin (3b) is $3.65 \mathrm{kcal} / \mathrm{mol}$. The energy difference between cinnafragrin A (2) conformer 1 and $\left(12^{\prime} R\right)$-capsicodendrin (3a) is $4.08 \mathrm{kcal} / \mathrm{mol}$. The energy difference between cinnafragrin A (2) conformer 2 and (12'S)-capsicodendrin (3b) is $3.29 \mathrm{kcal} / \mathrm{mol}$. The average value of these energy differences is $3.69 \mathrm{kcal} / \mathrm{mol}$.

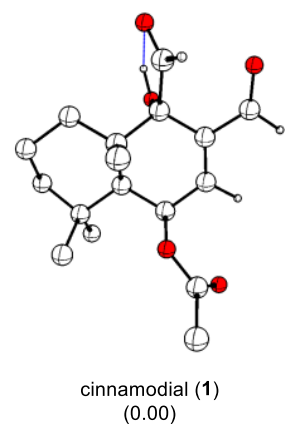

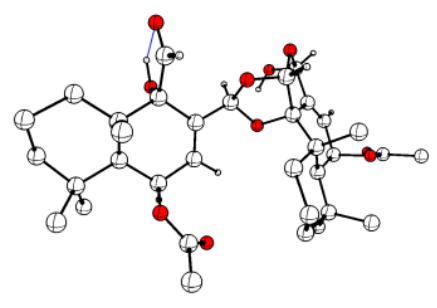

(12'S)-capsicodendrin (3a) (16.55)

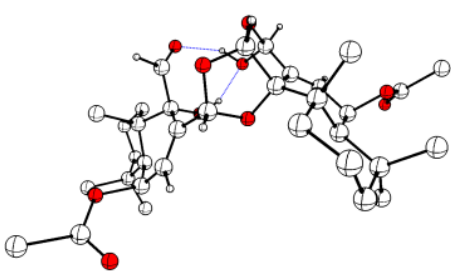

cinnafragrin A (2) conformer 1

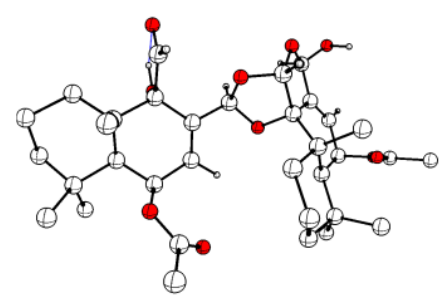

$\left(12^{\prime} R\right)$-capsicodendrin $(3 \mathbf{b})$

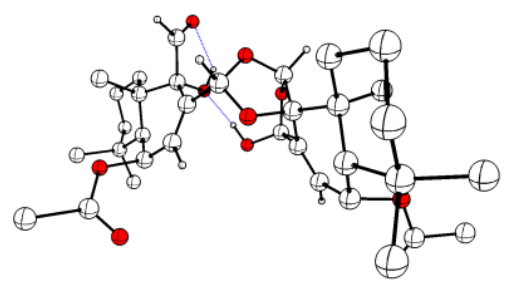

cinnafragrin A (2) conformer 2

Figure S4. Calculated structures and energies ( $\mathrm{kcal} / \mathrm{mol})$ of cinnamodial (1), cinnafragrin A (2), and capsicodendrin (3). 


\section{DFT Calculation Data}

DFT-optimized structure's energy components.

Table S6. Computed energy components for optimized structures.

\begin{tabular}{|c|c|c|c|c|}
\hline & $\mathrm{E}(\mathrm{SCF}) /(\mathrm{eV})$ & ZPE(kcal/mol) & $\mathrm{S}$ (gas) $/(\mathrm{cal} / \mathrm{mol} \cdot \mathrm{K})$ & $\begin{array}{c}\mathrm{G}(\text { solv }) \\
(\mathrm{kcal} / \mathrm{mol})\end{array}$ \\
\hline & $\begin{array}{l}\text { B3LYP-D3/ } \\
\text { cc-pVTZ(-f) }\end{array}$ & $\begin{array}{c}\text { B3LYP-D3/ } \\
6-31 \mathrm{G}^{* *}\end{array}$ & $\begin{array}{c}\text { B3LYP-D3/ } \\
6-31 \mathrm{G}^{* *}\end{array}$ & $\begin{array}{c}\text { B3LYP-D3/ } \\
6-31 G^{* *}\end{array}$ \\
\hline Cinnamodial (1) & -28266.336 & 245.589 & 151.04 & -14.52 \\
\hline $\begin{array}{c}\left(12{ }^{\prime} S\right)- \\
\text { capsicodendrin } \\
(\mathbf{3 a})\end{array}$ & -56533.215 & 496.64 & 245.41 & -22.33 \\
\hline $\begin{array}{c}\left(12^{\prime} R\right)- \\
\text { capsicodendrin } \\
(\mathbf{3 b})\end{array}$ & -56533.203 & 496.67 & 244.52 & -23.26 \\
\hline $\begin{array}{c}\text { Cinnafragrin A } \\
(\mathbf{2}) \\
\text { conformer } 1 \\
\end{array}$ & -56533.164 & 496.92 & 242.07 & -21.06 \\
\hline $\begin{array}{c}\text { Cinnafragrin A } \\
(\mathbf{2}) \\
\text { conformer } 2\end{array}$ & -56533.117 & 496.30 & 244.80 & -21.14 \\
\hline
\end{tabular}


Table S7. Cartesian coordinates of the optimized structures.

The Cartesian coordinates of optimizes geometries are given below in the standard XYZ format, and units are in $\AA$.

\begin{tabular}{|c|c|c|c|}
\hline \multicolumn{3}{|c|}{ namodial } & \multirow{2}{*}{$\begin{aligned}= & \\
& 5.919851303\end{aligned}$} \\
\hline $\mathrm{O}$ & 2.720479250 & 0.727414608 & \\
\hline $\mathrm{O}$ & -0.160304919 & 3.300086260 & 3.032307625 \\
\hline $\mathrm{O}$ & 4.382824898 & 2.418095827 & 7.034424305 \\
\hline $\mathrm{O}$ & 1.477367997 & 2.714090109 & 8.337257385 \\
\hline $\mathrm{C}$ & 1.661664844 & 1.660062075 & 3.331764698 \\
\hline $\mathrm{C}$ & 0.979981124 & 2.306986570 & 6.050697327 \\
\hline $\mathrm{C}$ & 2.439428806 & 2.098322392 & 5.683475971 \\
\hline $\mathrm{C}$ & 0.216956913 & 2.041796446 & 3.680089712 \\
\hline $\mathrm{C}$ & 1.933592200 & 1.449713588 & 1.795113921 \\
\hline $\mathrm{C}$ & 3.374698877 & 2.922720432 & 6.582014561 \\
\hline $\mathrm{C}$ & -0.010165384 & 2.228254557 & 5.151218891 \\
\hline $\mathrm{C}$ & 2.709480286 & 2.482120514 & 4.159351349 \\
\hline $\mathrm{C}$ & 4.143477440 & 2.019936323 & 3.802533627 \\
\hline $\mathrm{C}$ & 3.403313637 & 0.986344516 & 1.639651656 \\
\hline $\mathrm{C}$ & 0.643128216 & 2.524134398 & 7.468863964 \\
\hline $\mathrm{C}$ & 2.584335566 & 4.010752678 & 3.974409580 \\
\hline $\mathrm{C}$ & 1.697130799 & 2.669709921 & 0.875056267 \\
\hline $\mathrm{C}$ & 1.017107487 & 0.315702915 & 1.285083771 \\
\hline $\mathrm{C}$ & -1.712420106 & 4.812731743 & 2.111563444 \\
\hline $\mathrm{C}$ & 4.404144764 & 1.940763712 & 2.296017408 \\
\hline $\mathrm{C}$ & -1.485131145 & 3.508525610 & 2.835444927 \\
\hline $\mathrm{O}$ & -2.348560095 & 2.739906311 & 3.200337648 \\
\hline $\mathrm{H}$ & 3.556968451 & 0.728482604 & 6.423190117 \\
\hline $\mathrm{H}$ & 1.754790664 & 0.648016989 & 3.748611689 \\
\hline $\mathrm{H}$ & -0.475787044 & 1.276716113 & 3.319399357 \\
\hline $\mathrm{H}$ & 3.137786388 & 3.989750624 & 6.726859093 \\
\hline $\mathrm{H}$ & -1.049070239 & 2.335198402 & 5.456941128 \\
\hline $\mathrm{H}$ & 4.871861935 & 2.680639744 & 4.285701275 \\
\hline $\mathrm{H}$ & 4.296888828 & 1.021819592 & 4.224032879 \\
\hline $\mathrm{H}$ & 3.514523745 & -0.006633193 & 2.098101139 \\
\hline $\mathrm{H}$ & 3.625028372 & 0.862421989 & 0.572428226 \\
\hline
\end{tabular}

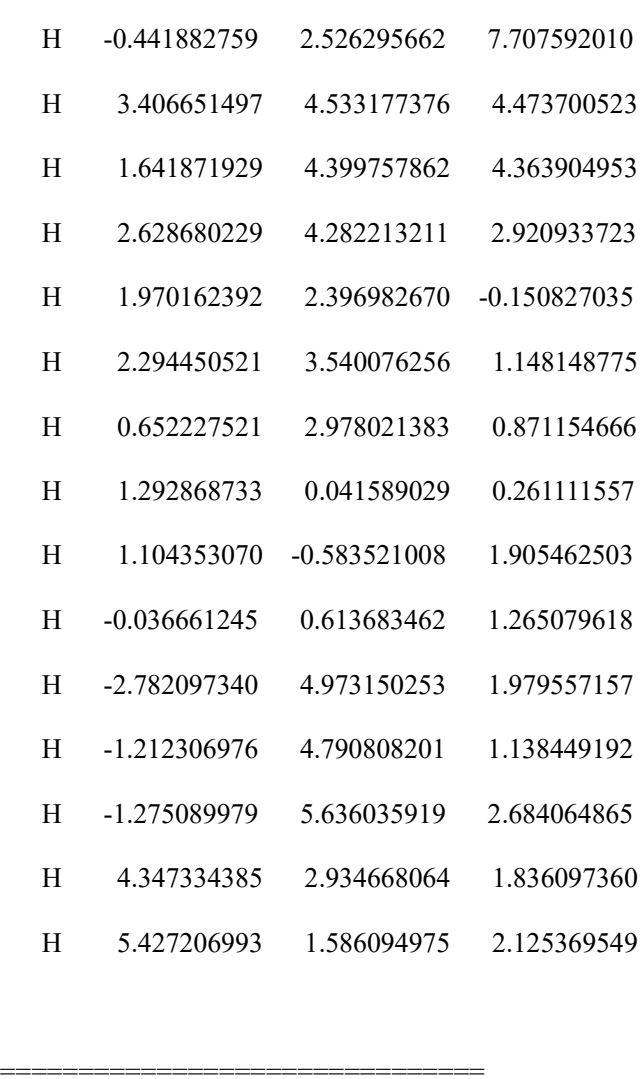

$(12 ' S)$-capsicodendrin

C $\quad-9.983843803 \quad-5.673786163 \quad-0.602930248$

C $\quad-10.212888718-5.710795403 \quad-2.116302967$

C $\quad-9.485200882-4.568552017 \quad-2.866556883$

C $\quad-9.925690651 \quad-3.206043243 \quad-2.208783627$

$\begin{array}{llll}\text { C } & -9.877158165 & -3.104092121 & -0.646861970\end{array}$

$\begin{array}{llll}\text { C } & -10.522704124 & -4.364382267 & -0.021888264\end{array}$

$\begin{array}{llll}\text { C } & -9.365693092 & -1.949183941 & -2.883821964\end{array}$

$\begin{array}{llll}\text { C } & -9.850504875 & -0.676783919 & -2.257072687\end{array}$

$\begin{array}{llll}\text { C } & -10.401548386 & -0.607587695 & -1.046294570\end{array}$

$\begin{array}{llll}\text { C } & -10.781537056 & -1.852993131 & -0.253733128\end{array}$

$\begin{array}{llll}\mathrm{H} & -10.999766350 & -3.146127939 & -2.434276819\end{array}$

$\begin{array}{llll}\text { C } & -10.669970512 & -1.633581758 & 1.263865829\end{array}$

H $\quad-9.705262184 \quad-1.291002512 \quad 1.664138675$

$\begin{array}{llll}\text { O } & -11.619613647 & -1.866850853 & 1.985611558\end{array}$

$\begin{array}{llll}\text { O } & -12.152299881 & -2.085194826 & -0.549656510\end{array}$ 


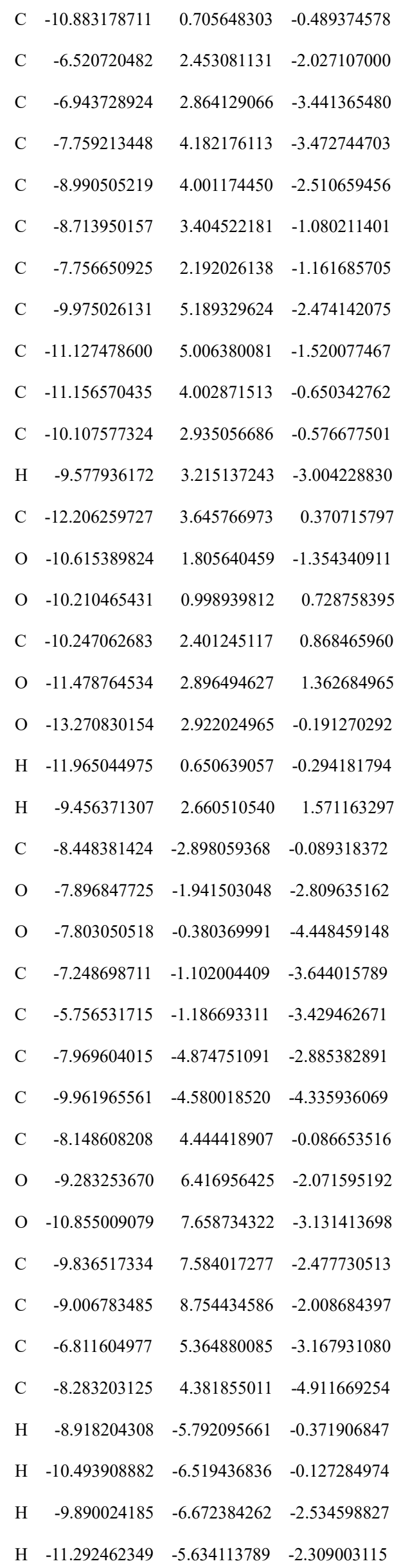

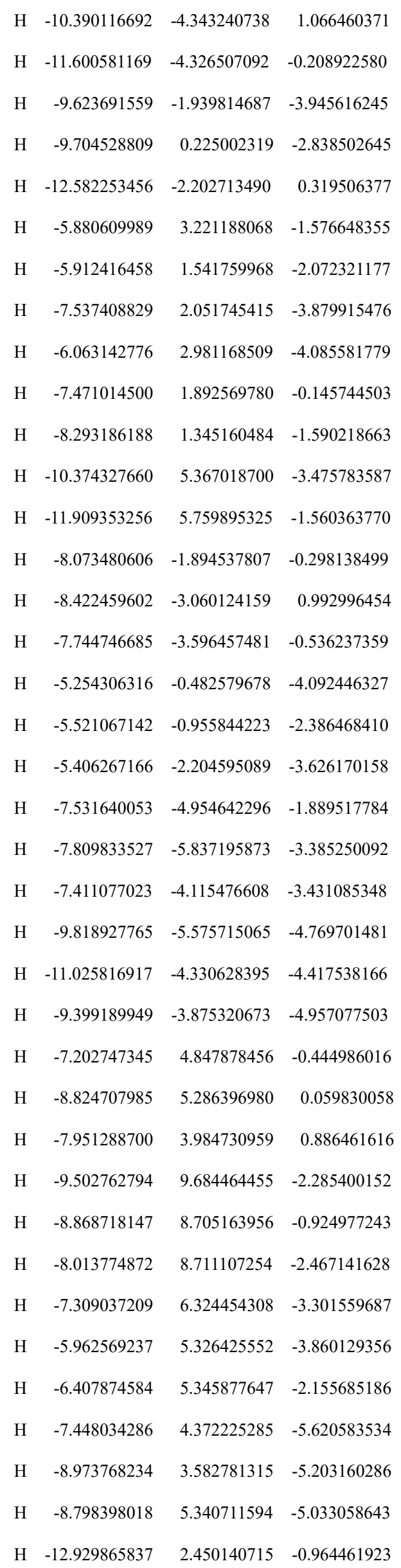




\section{$\begin{array}{llll}\mathrm{H} & -12.653744698 & 4.494431019 & 0.893264055\end{array}$}

$\left(12^{\prime} R\right)$-capsicodendrin
$\begin{array}{llll}\text { C } & -10.142906189 & -5.716581821 & -0.737848818\end{array}$
$\begin{array}{llll}\text { C } & -10.293666840 & -5.716132164 & -2.261831284\end{array}$
$\begin{array}{llll}\text { C } & -9.496946335 & -4.579683781 & -2.949479103\end{array}$
$\begin{array}{llll}\text { C } & -9.927955627 & -3.218726158 & -2.282681465\end{array}$
$\begin{array}{llll}\text { C } & -9.958532333 & -3.150769949 & -0.718342960\end{array}$
$\begin{array}{llll}\text { C } & -10.672514915 & -4.403415203 & -0.156333834\end{array}$
$\begin{array}{llll}\text { C } & -9.301755905 & -1.964180827 & -2.901988029\end{array}$
$\begin{array}{llll}\text { C } & -9.781529427 & -0.693785131 & -2.270424366\end{array}$
$\begin{array}{llll}\text { C } & -10.390833855 & -0.632999182 & -1.087640524\end{array}$
$\begin{array}{llll}\text { C } & -10.848208427 & -1.882308960 & -0.344267190\end{array}$
H $\quad-10.986945152 \quad-3.121550798 \quad-2.558986664$
$\begin{array}{llll}\text { C } & -10.812978745 & -1.689859271 & 1.180201650\end{array}$
$\begin{array}{llll}\mathrm{H} & -9.860738754 & -1.391418576 & 1.640166521\end{array}$
$\begin{array}{llll}\text { O } & -11.812191010 & -1.889099002 & 1.843839169\end{array}$
$\begin{array}{llll}\text { O } & -12.206974983 & -2.071267366 & -0.716080427\end{array}$
$\begin{array}{llll}\text { C } & -10.822416306 & 0.695592403 & -0.520116985\end{array}$
$\begin{array}{llll}\text { C } & -6.439461708 & 2.564256668 & -2.069961786\end{array}$
$\begin{array}{llll}\text { C } & -6.911368370 & 2.963563442 & -3.472311974\end{array}$
$\begin{array}{llll}\text { C } & -7.775483608 & 4.251441956 & -3.477061033\end{array}$
$\begin{array}{llll}\text { C } & -8.985685349 & 4.000521183 & -2.506789207\end{array}$
$\begin{array}{llll}\text { C } & -8.653706551 & 3.435844898 & -1.077006578\end{array}$
$\begin{array}{llll}\text { C } & -7.638465405 & 2.269078970 & -1.162758589\end{array}$
$\begin{array}{llll}\text { C } & -10.055250168 & 5.117159367 & -2.476328135\end{array}$
$\begin{array}{llll}\text { C } & -11.144342422 & 4.913198948 & -1.454112411\end{array}$
$\begin{array}{llll}\text { C } & -11.099655151 & 3.919658899 & -0.572934926\end{array}$
$\begin{array}{llll}\text { C } & -10.013852119 & 2.890766144 & -0.551792562\end{array}$
H $\quad-9.520840645 \quad 3.172479630 \quad-2.989449739$
$\begin{array}{llll}\text { C } & -12.039942741 & 3.649493933 & 0.575807691\end{array}$
$\begin{array}{llll}\text { O } & -10.454611778 & 1.771048546 & -1.358027339\end{array}$
$\begin{array}{llll}\mathrm{O} & -10.154288292 & 0.925593138 & 0.719873369\end{array}$
$\begin{array}{llll}\text { C } & -10.088054657 & 2.324335814 & 0.892617583\end{array}$
$\begin{array}{llll}\text { O } & -11.240216255 & 2.874656916 & 1.498389244\end{array}$
$\begin{array}{llll}\text { O } & -12.543885231 & 4.784359455 & 1.197765708\end{array}$

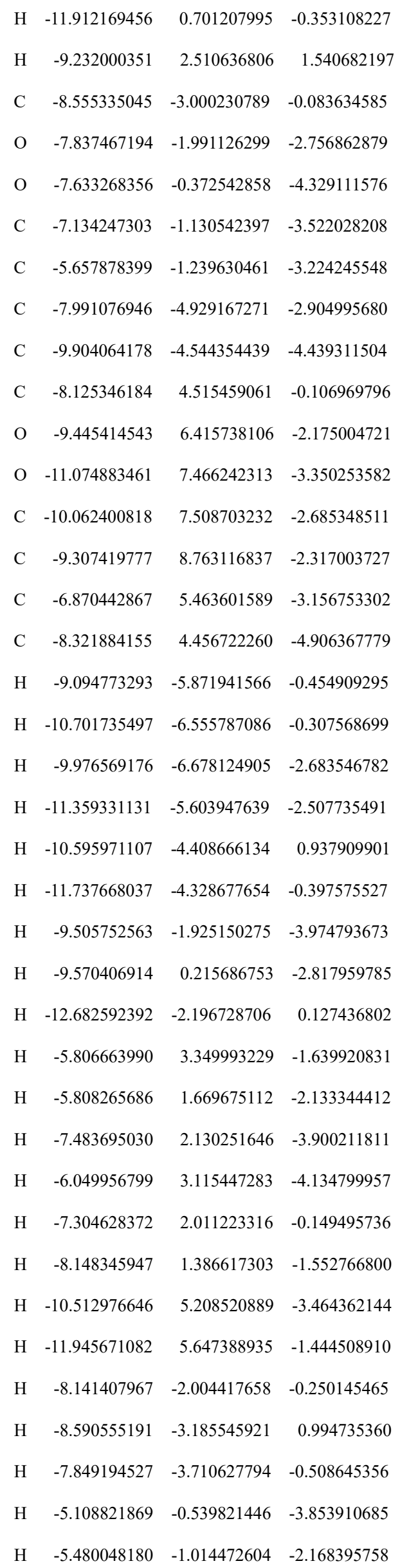




\begin{tabular}{llll}
$\mathrm{H}$ & -5.310802937 & -2.261624098 & -3.403830528 \\
$\mathrm{H}$ & -7.603724957 & -5.043010712 & -1.891554832 \\
$\mathrm{H}$ & -7.834991455 & -5.885129452 & -3.418339014 \\
$\mathrm{H}$ & -7.385146618 & -4.175115108 & -3.406475306 \\
$\mathrm{H}$ & -9.768471718 & -5.533959389 & -4.889025211 \\
$\mathrm{H}$ & -10.955590248 & -4.262576580 & -4.564583302 \\
$\mathrm{H}$ & -9.293310165 & -3.842436552 & -5.016703606 \\
$\mathrm{H}$ & -7.140870094 & 4.864396572 & -0.418225080 \\
$\mathrm{H}$ & -8.779148102 & 5.385982037 & -0.059089653 \\
$\mathrm{H}$ & -8.011096001 & 4.112204552 & 0.904004037 \\
$\mathrm{H}$ & -9.866704941 & 9.635008812 & -2.655408382 \\
$\mathrm{H}$ & -9.152179718 & 8.806175232 & -1.235488892 \\
$\mathrm{H}$ & -8.319653511 & 8.750590324 & -2.788815975 \\
$\mathrm{H}$ & -7.403512955 & 6.405650616 & -3.274638891 \\
$\mathrm{H}$ & -6.022389412 & 5.466556549 & -3.851398230 \\
$\mathrm{H}$ & -6.463091373 & 5.445463657 & -2.145717382 \\
$\mathrm{H}$ & -7.494790554 & 4.494359970 & -5.623768806 \\
$\mathrm{H}$ & -8.983121872 & 3.636062622 & -5.206011295 \\
$\mathrm{H}$ & -8.877717018 & 5.395588875 & -5.003030300 \\
$\mathrm{H}$ & -11.790689468 & 5.357439995 & 1.403403997 \\
$\mathrm{H}$ & -12.919692039 & 3.062517881 & 0.284727603 \\
\hline
\end{tabular}

cinnafragrin A conformer 1

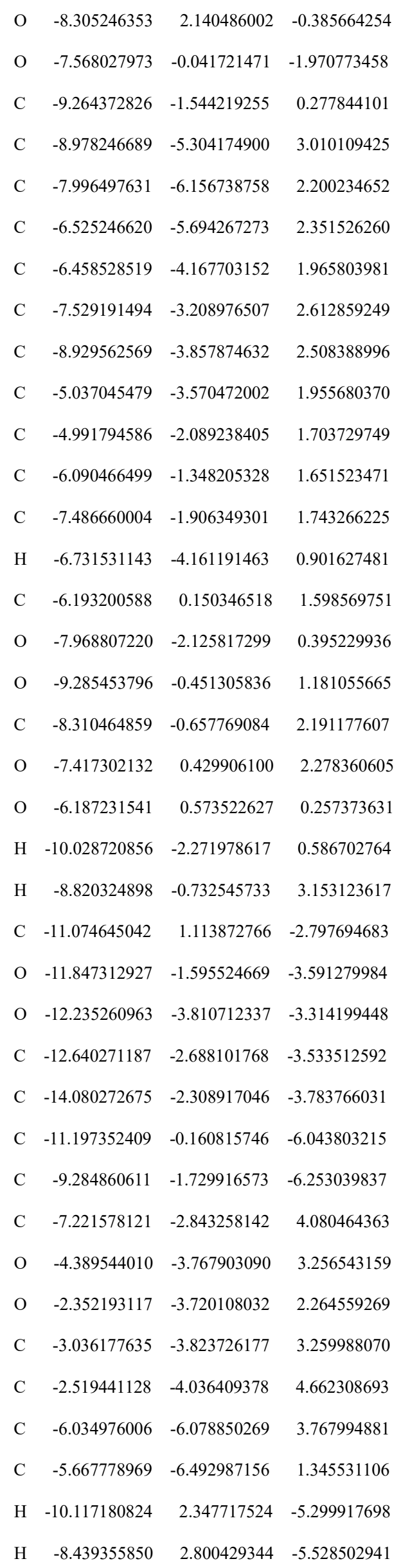




\begin{tabular}{|c|c|c|c|c|c|c|c|}
\hline $\mathrm{H}$ & -8.971307755 & 0.897120059 & -7.035508633 & $\mathrm{H}$ & -4.598773003 & -6.286822796 & 1.461638808 \\
\hline $\mathrm{H}$ & -7.794082165 & 0.412828594 & -5.819612503 & $\mathrm{H}$ & -6.689183235 & 1.409130573 & 0.161292374 \\
\hline $\mathrm{H}$ & -8.937191963 & 2.666165590 & -3.095046520 & $\mathrm{H}$ & -5.409668446 & 0.670174658 & 2.163431168 \\
\hline $\mathrm{H}$ & -7.731285572 & 1.488651037 & -3.576159477 & & & & \\
\hline $\mathrm{H}$ & -10.162022591 & -2.749569178 & -3.897696972 & & & & \\
\hline $\mathrm{H}$ & -10.555261612 & -2.931167364 & -1.507597566 & \multirow{2}{*}{\multicolumn{3}{|c|}{ cinnafragrin A conformer 2}} & \\
\hline $\mathrm{H}$ & -7.067633152 & -0.134138018 & -1.135198832 & & & & \\
\hline $\mathrm{H}$ & -8.748542786 & -5.360928059 & 4.080327034 & $\mathrm{C}$ & -8.598736763 & 1.800495148 & -5.066389084 \\
\hline $\mathrm{H}$ & -9.996411324 & -5.693449974 & 2.895110607 & $\mathrm{C}$ & -8.359542847 & 0.493710786 & -5.828024387 \\
\hline $\mathrm{H}$ & -8.281689644 & -6.106239796 & 1.139550328 & C & -9.396496773 & -0.603256226 & -5.481308937 \\
\hline $\mathrm{H}$ & -8.068073273 & -7.212146282 & 2.490069866 & $\mathrm{C}$ & -9.372676849 & -0.800075114 & -3.918315887 \\
\hline $\mathrm{H}$ & -9.662511826 & -3.232478142 & 3.034620285 & C & -9.446959496 & 0.482641488 & -3.021327496 \\
\hline $\mathrm{H}$ & -9.220263481 & -3.882448196 & 1.454452634 & $\mathrm{C}$ & -8.475272179 & 1.563420773 & -3.558309317 \\
\hline $\mathrm{H}$ & -4.422800064 & -4.085706234 & 1.213586688 & $\mathrm{C}$ & -10.270631790 & -1.924957275 & -3.389644861 \\
\hline $\mathrm{H}$ & -4.001152515 & -1.645831585 & 1.649535060 & $\mathrm{C}$ & -10.247648239 & -2.069813490 & -1.897813082 \\
\hline $\mathrm{H}$ & -11.638967514 & 0.435890138 & -2.155739546 & $\mathrm{C}$ & -9.702227592 & -1.185516000 & -1.065776587 \\
\hline $\mathrm{H}$ & -11.065399170 & 2.105354309 & -2.334459543 & $\mathrm{C}$ & -8.930430412 & 0.024261083 & -1.583562970 \\
\hline $\mathrm{H}$ & -11.629831314 & 1.200136304 & -3.729429007 & $\mathrm{H}$ & -8.361776352 & -1.186104178 & -3.732065678 \\
\hline $\mathrm{H}$ & -14.713057518 & -3.189011097 & -3.671582937 & $\mathrm{C}$ & -9.013072014 & 1.212574124 & -0.623532355 \\
\hline $\mathrm{H}$ & -14.387844086 & -1.527926326 & -3.082593203 & $\mathrm{H}$ & -10.019056320 & 1.496930242 & -0.263844997 \\
\hline $\mathrm{H}$ & -14.184864998 & -1.900157332 & -4.793701649 & $\mathrm{O}$ & -8.022665024 & 1.835945845 & -0.303615600 \\
\hline $\mathrm{H}$ & -11.639102936 & 0.736487985 & -5.608565331 & $\mathrm{O}$ & -7.549653530 & -0.345232785 & -1.669324994 \\
\hline $\mathrm{H}$ & -11.162770271 & -0.011201231 & -7.129232407 & $\mathrm{C}$ & -9.805630684 & -1.397245526 & 0.439731538 \\
\hline $\mathrm{H}$ & -11.874542236 & -0.990486383 & -5.842239857 & C & -11.043243408 & -3.467555761 & 4.695632935 \\
\hline $\mathrm{H}$ & -9.253048897 & -1.564053893 & -7.335519314 & $\mathrm{C}$ & -11.795115471 & -4.681317329 & 4.137973785 \\
\hline $\mathrm{H}$ & -8.278477669 & -2.019365311 & -5.930409908 & $\mathrm{C}$ & -10.852306366 & -5.788938522 & 3.600617409 \\
\hline $\mathrm{H}$ & -9.954928398 & -2.578246117 & -6.077380180 & C & -9.913124084 & -5.125236511 & 2.526691437 \\
\hline $\mathrm{H}$ & -7.251663685 & -3.725517750 & 4.718428612 & C & -9.201222420 & -3.778668404 & 2.923665285 \\
\hline $\mathrm{H}$ & -6.234377861 & -2.394998550 & 4.190989971 & C & -10.211022377 & -2.812278986 & 3.588832140 \\
\hline $\mathrm{H}$ & -7.965174198 & -2.142989159 & 4.473304272 & C & -8.974805832 & -6.100680828 & 1.788090944 \\
\hline $\mathrm{H}$ & -1.430085063 & -4.008679390 & 4.655138493 & $\mathrm{C}$ & -8.014720917 & -5.436325550 & 0.837683916 \\
\hline $\mathrm{H}$ & -2.915031672 & -3.264481544 & 5.328327179 & $\mathrm{C}$ & -7.890069962 & -4.116943359 & 0.763633788 \\
\hline $\mathrm{H}$ & -2.864515543 & -5.003501415 & 5.041616440 & $\mathrm{C}$ & -8.731776237 & -3.163723230 & 1.567957997 \\
\hline $\mathrm{H}$ & -4.964369297 & -5.917886734 & 3.883236170 & $\mathrm{H}$ & -10.613141060 & -4.818877697 & 1.737962246 \\
\hline $\mathrm{H}$ & -6.232890606 & -7.144351482 & 3.932204008 & $\mathrm{C}$ & -6.867888451 & -3.313697338 & -0.004388177 \\
\hline $\mathrm{H}$ & -6.533494473 & -5.527951717 & 4.565502167 & $\mathrm{O}$ & -9.887208939 & -2.762079716 & 0.789245546 \\
\hline $\mathrm{H}$ & -5.807988167 & -7.568147659 & 1.502091289 & $\mathrm{O}$ & -8.711511612 & -0.842243254 & 1.154782176 \\
\hline $\mathrm{H}$ & -5.946383953 & -6.268153191 & 0.309850454 & $\mathrm{C}$ & -7.862512589 & -1.879880428 & 1.597852588 \\
\hline
\end{tabular}




\begin{tabular}{|c|c|c|c|c|c|c|c|}
\hline $\mathrm{O}$ & -6.738258839 & -2.109271288 & 0.769616961 & $\mathrm{H}$ & -14.517015457 & -3.228117943 & -4.271624088 \\
\hline $\mathrm{O}$ & -7.277684212 & -3.063922644 & -1.316095233 & $\mathrm{H}$ & -14.251934052 & -1.590782762 & -3.593219280 \\
\hline $\mathrm{H}$ & -10.709513664 & -0.890242636 & 0.814431429 & $\mathrm{H}$ & -13.833934784 & -1.915297747 & -5.275031090 \\
\hline $\mathrm{H}$ & -7.487608910 & -1.566500545 & 2.571672916 & $\mathrm{H}$ & -11.172031403 & 0.705966353 & -5.727150440 \\
\hline $\mathrm{C}$ & -10.871874809 & 1.067770123 & -2.897166252 & $\mathrm{H}$ & -10.606929779 & -0.079412252 & -7.197123051 \\
\hline $\mathrm{O}$ & -11.667029381 & -1.681482077 & -3.769765139 & $\mathrm{H}$ & -11.498917580 & -1.002005816 & -5.982846260 \\
\hline $\mathrm{O}$ & -12.112471581 & -3.899428368 & -3.623194218 & $\mathrm{H}$ & -8.799855232 & -1.771024585 & -7.221032619 \\
\hline $\mathrm{C}$ & -12.473505020 & -2.765401602 & -3.852004528 & $\mathrm{H}$ & -7.995309353 & -2.279994249 & -5.729017258 \\
\hline $\mathrm{C}$ & -13.864881516 & -2.355067730 & -4.273073196 & $\mathrm{H}$ & -9.686349869 & -2.717800140 & -6.027561188 \\
\hline $\mathrm{C}$ & -10.752433777 & -0.220988557 & -6.119803905 & $\mathrm{H}$ & -8.274689674 & -4.428354740 & 4.792417049 \\
\hline C & -8.943498611 & -1.922134519 & -6.145511627 & $\mathrm{H}$ & -7.231527328 & -4.634697437 & 3.395260334 \\
\hline $\mathrm{C}$ & -7.978977203 & -3.981292009 & 3.844444036 & $\mathrm{H}$ & -7.504740715 & -3.023592949 & 4.080163002 \\
\hline $\mathrm{O}$ & -8.146659851 & -6.831943989 & 2.753529787 & $\mathrm{H}$ & -6.487245083 & -9.636752129 & 3.135258436 \\
\hline $\mathrm{O}$ & -7.981472015 & -8.579541206 & 1.319242239 & $\mathrm{H}$ & -6.062276840 & -8.007109642 & 3.748040438 \\
\hline $\mathrm{C}$ & -7.717414856 & -8.060640335 & 2.382601023 & $\mathrm{H}$ & -7.492527008 & -8.827405930 & 4.372516155 \\
\hline $\mathrm{C}$ & -6.880822659 & -8.679695129 & 3.476817608 & $\mathrm{H}$ & -9.586547852 & -7.347533703 & 4.493488789 \\
\hline $\mathrm{C}$ & -10.166161537 & -6.479162693 & 4.802937984 & $\mathrm{H}$ & -10.938645363 & -6.822226048 & 5.501063347 \\
\hline $\mathrm{C}$ & -11.718497276 & -6.859369278 & 2.901458025 & $\mathrm{H}$ & -9.496297836 & -5.821867466 & 5.357188225 \\
\hline $\mathrm{H}$ & -9.582108498 & 2.216157913 & -5.317049026 & $\mathrm{H}$ & -12.482967377 & -7.235426426 & 3.590315580 \\
\hline $\mathrm{H}$ & -7.863439560 & 2.553323269 & -5.373436451 & $\mathrm{H}$ & -12.232325554 & -6.452462196 & 2.023175955 \\
\hline $\mathrm{H}$ & -8.373991013 & 0.667529881 & -6.911188126 & $\mathrm{H}$ & -11.124125481 & -7.719789505 & 2.577205420 \\
\hline H & -7.352976799 & 0.124446385 & -5.584946156 & $\mathrm{H}$ & -7.233007431 & -2.097407341 & -1.465493321 \\
\hline $\mathrm{H}$ & -8.629064560 & 2.500484467 & -3.010515213 & $\mathrm{H}$ & -5.875103951 & -3.786115170 & 0.001263185 \\
\hline $\mathrm{H}$ & -7.450690746 & 1.242154837 & -3.349052191 & & & & \\
\hline $\mathrm{H}$ & -9.979421616 & -2.878654242 & -3.834590912 & & & & \\
\hline $\mathrm{H}$ & -10.720982552 & -2.963402271 & -1.504421473 & & & & \\
\hline $\mathrm{H}$ & -7.066319942 & 0.282582134 & -1.100122452 & & & & \\
\hline $\mathrm{H}$ & -10.409374237 & -3.764530420 & 5.539422035 & & & & \\
\hline $\mathrm{H}$ & -11.756757736 & -2.736954689 & 5.094501495 & & & & \\
\hline $\mathrm{H}$ & -12.450112343 & -4.343071461 & 3.321974993 & & & & \\
\hline $\mathrm{H}$ & -12.451442719 & -5.114296913 & 4.903105259 & & & & \\
\hline $\mathrm{H}$ & -9.673772812 & -1.934844375 & 3.970379829 & & & & \\
\hline $\mathrm{H}$ & -10.903077126 & -2.451053143 & 2.821517467 & & & & \\
\hline $\mathrm{H}$ & -9.563802719 & -6.845661163 & 1.248122096 & & & & \\
\hline $\mathrm{H}$ & -7.378047943 & -6.103738785 & 0.262697160 & & & & \\
\hline $\mathrm{H}$ & -11.549975395 & 0.388009667 & -2.379350662 & & & & \\
\hline $\mathrm{H}$ & -10.858442307 & 2.023893118 & -2.363229990 & & & & \\
\hline $\mathrm{H}$ & -11.301918983 & 1.258810639 & -3.878859520 & & & & \\
\hline
\end{tabular}


Table S8. Vibrational frequencies $\left(\right.$ in $\mathrm{cm}^{-1}$ ) of the optimized structures.

cinnamodial

\begin{tabular}{rrrrrr}
35.95 & 56.42 & 60.80 & 64.11 & 86.30 & 104.51 \\
128.67 & 132.61 & 162.50 & 165.17 & 192.20 & 206.02 \\
207.92 & 236.52 & 240.49 & 262.99 & 278.42 & 295.08 \\
302.45 & 309.01 & 314.49 & 329.34 & 343.92 & 359.05 \\
369.55 & 389.37 & 397.56 & 440.26 & 454.30 & 468.22 \\
482.59 & 489.14 & 509.86 & 540.07 & 561.24 & 591.42 \\
604.00 & 612.19 & 644.32 & 665.01 & 706.54 & 727.76 \\
751.60 & 799.47 & 822.20 & 864.80 & 887.98 & 898.56 \\
\hline 906.78 & 915.53 & 945.21 & 948.05 & 957.04 & 967.71 \\
984.16 & 1006.84 & 1009.65 & 1020.49 & 1033.11 & 1036.70 \\
1050.74 & 1065.53 & 1067.06 & 1070.40 & 1083.12 & 1088.42 \\
1133.14 & 1137.98 & 1157.21 & 1197.37 & 1209.29 & 1223.74 \\
\hline 1229.22 & 1241.80 & 1246.10 & 1270.64 & 1299.26 & 1339.43 \\
\hline 1354.53 & 1369.22 & 1371.03 & 1381.07 & 1384.16 & 1387.07 \\
\hline 1397.47 & 1399.81 & 1409.33 & 1411.73 & 1420.87 & 1423.10 \\
\hline 1433.61 & 1443.54 & 1450.48 & 1480.30 & 1487.42 & 1494.81 \\
\hline 1499.21 & 1503.10 & 1514.06 & 1519.17 & 1521.70 & 1525.54 \\
1531.37 & 1537.73 & 1724.11 & 1781.05 & 1800.60 & 1824.83 \\
\hline 2924.85 & 3011.84 & 3017.83 & 3031.62 & 3035.75 & 3042.69 \\
\hline 3053.84 & 3055.25 & 3063.69 & 3064.75 & 3067.87 & 3079.80 \\
3092.86 & 3105.59 & 3107.37 & 3108.21 & 3131.23 & 3134.72 \\
3139.78 & 3168.83 & 3176.24 & 3178.16 & 3180.75 & 3641.42
\end{tabular}

(12'S)-capsicodendrin

$\begin{array}{rrrrrr}14.97 & 23.93 & 26.73 & 43.23 & 50.51 & 58.12 \\ 59.53 & 63.75 & 65.07 & 80.06 & 87.57 & 91.85 \\ 93.70 & 106.55 & 108.99 & 112.64 & 116.42 & 128.53 \\ 130.94 & 137.04 & 155.56 & 166.75 & 167.69 & 199.38 \\ 201.75 & 205.00 & 206.84 & 210.98 & 227.28 & 235.09 \\ 241.06 & 255.41 & 262.99 & 275.75 & 276.80 & 287.88 \\ 291.16 & 300.34 & 303.49 & 307.02 & 311.44 & 313.23 \\ 319.19 & 327.56 & 331.35 & 335.76 & 342.71 & 353.91\end{array}$




\begin{tabular}{|c|c|c|c|c|c|}
\hline 362.55 & 369.30 & 388.11 & 393.86 & 403.41 & 409.44 \\
\hline 414.14 & 439.61 & 441.22 & 448.29 & 456.96 & 461.09 \\
\hline 471.23 & 474.08 & 479.34 & 499.92 & 503.18 & 509.34 \\
\hline 515.69 & 525.55 & 541.10 & 544.84 & 570.44 & 575.25 \\
\hline 585.15 & 592.87 & 604.40 & 606.91 & 611.65 & 646.67 \\
\hline 655.79 & 668.98 & 675.82 & 688.04 & 721.39 & 732.04 \\
\hline 743.12 & 750.74 & 752.42 & 770.13 & 781.92 & 796.36 \\
\hline 802.75 & 825.70 & 858.75 & 867.94 & 877.46 & 883.33 \\
\hline 888.34 & 892.09 & 898.27 & 902.96 & 915.79 & 916.42 \\
\hline 941.38 & 942.34 & 947.37 & 949.19 & 951.00 & 953.24 \\
\hline 958.23 & 964.91 & 965.94 & 973.76 & 984.54 & 986.06 \\
\hline 997.02 & 1001.64 & 1005.61 & 1008.59 & 1011.61 & 1012.25 \\
\hline 1019.89 & 1027.01 & 1035.90 & 1036.45 & 1044.30 & 1048.25 \\
\hline 1057.55 & 1061.65 & 1065.87 & 1067.24 & 1071.09 & 1074.72 \\
\hline 1078.44 & 1082.63 & 1086.21 & 1090.62 & 1095.23 & 1108.47 \\
\hline 1125.91 & 1132.79 & 1136.72 & 1151.61 & 1154.92 & 1168.04 \\
\hline 1197.02 & 1200.58 & 1206.80 & 1209.53 & 1220.10 & 1221.86 \\
\hline 1226.46 & 1228.84 & 1240.78 & 1241.47 & 1246.24 & 1250.67 \\
\hline 1269.08 & 1274.46 & 1279.33 & 1295.25 & 1296.99 & 1325.34 \\
\hline 1336.72 & 1338.79 & 1346.28 & 1353.81 & 1360.54 & 1362.74 \\
\hline 1369.50 & 1372.29 & 1374.33 & 1378.72 & 1379.83 & 1381.29 \\
\hline 1382.87 & 1384.97 & 1386.00 & 1389.92 & 1394.41 & 1395.40 \\
\hline 1400.27 & 1401.91 & 1408.26 & 1411.44 & 1411.60 & 1414.03 \\
\hline 1421.52 & 1422.50 & 1425.60 & 1435.44 & 1441.72 & 1442.85 \\
\hline 1449.12 & 1450.26 & 1451.11 & 1479.46 & 1480.31 & 1485.91 \\
\hline 1488.11 & 1494.59 & 1495.78 & 1498.81 & 1499.98 & 1501.20 \\
\hline 1504.89 & 1513.36 & 1515.47 & 1517.89 & 1518.73 & 1521.89 \\
\hline 1521.98 & 1525.86 & 1526.50 & 1531.57 & 1532.00 & 1536.92 \\
\hline 1537.66 & 1758.60 & 1785.54 & 1800.08 & 1810.21 & 1823.22 \\
\hline 3010.65 & 3017.36 & 3026.31 & 3029.51 & 3032.02 & 3035.94 \\
\hline 3036.47 & 3040.09 & 3040.35 & 3046.64 & 3050.89 & 3051.95 \\
\hline 3053.58 & 3056.18 & 3063.04 & 3063.57 & 3065.75 & 3067.76 \\
\hline 3070.97 & 3071.70 & 3078.21 & 3083.00 & 3097.32 & 3097.86 \\
\hline 3100.70 & 3106.76 & 3106.85 & 3110.53 & 3110.99 & 3112.77 \\
\hline 3130.97 & 3131.76 & 3131.89 & 3138.21 & 3139.11 & 3147.59 \\
\hline 3150.67 & 3154.90 & 3172.30 & 3173.50 & 3178.91 & 3179.83 \\
\hline 3182.86 & 3189.18 & 3193.92 & 3234.35 & 3631.29 & 3793.16 \\
\hline
\end{tabular}


$\left(12^{\prime} R\right)$-capsicodendrin

\begin{tabular}{|c|c|c|c|c|c|}
\hline 22.70 & 27.75 & 31.22 & 42.13 & 48.38 & 53.99 \\
\hline 62.19 & 67.62 & 68.66 & 76.97 & 83.79 & 88.70 \\
\hline 95.16 & 101.57 & 107.59 & 112.15 & 113.29 & 127.34 \\
\hline 135.41 & 137.28 & 154.62 & 158.44 & 166.84 & 198.69 \\
\hline 201.42 & 203.71 & 210.19 & 213.21 & 228.41 & 235.29 \\
\hline 244.25 & 254.83 & 271.93 & 275.50 & 283.13 & 289.17 \\
\hline 294.64 & 299.79 & 304.96 & 306.64 & 312.00 & 314.10 \\
\hline 318.97 & 327.16 & 330.07 & 337.35 & 346.28 & 353.00 \\
\hline 360.03 & 366.22 & 370.72 & 388.78 & 396.15 & 398.33 \\
\hline 411.71 & 422.20 & 428.46 & 445.70 & 451.01 & 457.98 \\
\hline 468.86 & 474.73 & 478.47 & 492.30 & 499.49 & 508.23 \\
\hline 514.64 & 523.95 & 532.73 & 567.56 & 573.27 & 576.41 \\
\hline 596.27 & 603.44 & 604.94 & 610.28 & 622.44 & 636.01 \\
\hline 656.26 & 668.33 & 676.69 & 681.25 & 720.53 & 733.32 \\
\hline 740.52 & 750.61 & 752.46 & 772.68 & 783.31 & 797.91 \\
\hline 803.57 & 826.13 & 850.50 & 861.90 & 874.87 & 883.95 \\
\hline 888.56 & 897.57 & 900.21 & 902.78 & 912.22 & 916.55 \\
\hline 937.16 & 944.42 & 947.97 & 950.41 & 952.18 & 955.65 \\
\hline 961.67 & 963.21 & 966.53 & 983.63 & 986.56 & 988.21 \\
\hline 994.71 & 1004.42 & 1008.11 & 1008.91 & 1010.72 & 1015.35 \\
\hline 1020.79 & 1029.44 & 1036.83 & 1038.20 & 1046.68 & 1053.92 \\
\hline 1060.96 & 1066.16 & 1066.83 & 1068.46 & 1072.66 & 1077.99 \\
\hline 1081.48 & 1084.49 & 1088.84 & 1094.01 & 1115.70 & 1120.86 \\
\hline 1130.39 & 1136.34 & 1147.85 & 1152.09 & 1155.36 & 1169.00 \\
\hline 1196.90 & 1202.99 & 1206.87 & 1209.79 & 1218.95 & 1223.72 \\
\hline 1227.18 & 1229.08 & 1240.99 & 1243.22 & 1246.14 & 1254.67 \\
\hline 1272.69 & 1278.97 & 1294.93 & 1296.96 & 1305.24 & 1322.59 \\
\hline 1333.65 & 1336.81 & 1339.43 & 1348.56 & 1354.52 & 1361.09 \\
\hline 1361.97 & 1366.72 & 1374.07 & 1378.04 & 1380.22 & 1380.83 \\
\hline 1382.89 & 1385.65 & 1386.55 & 1389.72 & 1394.45 & 1397.49 \\
\hline 1400.32 & 1401.84 & 1408.73 & 1411.83 & 1412.12 & 1413.92 \\
\hline 1421.75 & 1422.71 & 1425.62 & 1433.25 & 1442.63 & 1443.98 \\
\hline 1449.90 & 1450.67 & 1456.79 & 1479.39 & 1480.63 & 1486.45 \\
\hline 1489.10 & 1494.86 & 1496.35 & 1500.09 & 1500.45 & 1501.95 \\
\hline
\end{tabular}




$\begin{array}{llllll}1505.19 & 1513.57 & 1515.07 & 1519.23 & 1520.02 & 1522.28 \\ 1522.36 & 1525.84 & 1526.63 & 1532.07 & 1532.10 & 1538.09 \\ 1538.54 & 1758.92 & 1781.53 & 1798.06 & 1811.21 & 1825.45 \\ 2989.31 & 3018.00 & 3029.27 & 3032.68 & 3034.86 & 3036.53 \\ 3037.40 & 3038.86 & 3039.31 & 3046.15 & 3047.62 & 3052.93 \\ 3055.52 & 3056.41 & 3056.65 & 3063.54 & 3064.90 & 3066.56 \\ 3069.53 & 3070.61 & 3071.30 & 3078.30 & 3083.32 & 3098.50 \\ 3101.02 & 3103.76 & 3107.43 & 3108.08 & 3111.92 & 3112.26 \\ 3130.94 & 3132.19 & 3132.90 & 3136.96 & 3139.14 & 3140.89 \\ 3142.67 & 3151.08 & 3169.21 & 3175.22 & 3178.84 & 3180.67 \\ 3182.63 & 3185.16 & 3195.51 & 3241.16 & 3635.82 & 3786.34\end{array}$

cinnafragrin A conformer 1

\begin{tabular}{|c|c|c|c|c|c|}
\hline 20.72 & 24.18 & 34.31 & 43.56 & 48.64 & 52.73 \\
\hline 58.20 & 61.24 & 65.19 & 76.64 & 79.85 & 93.50 \\
\hline 97.00 & 108.55 & 121.62 & 123.30 & 134.47 & 141.04 \\
\hline 149.18 & 158.43 & 164.96 & 173.80 & 182.37 & 191.88 \\
\hline 202.47 & 208.86 & 214.74 & 218.88 & 225.46 & 237.20 \\
\hline 247.24 & 252.72 & 260.08 & 284.76 & 284.90 & 290.62 \\
\hline 298.19 & 300.35 & 307.70 & 309.63 & 313.21 & 318.78 \\
\hline 321.96 & 323.25 & 328.62 & 334.79 & 347.62 & 357.89 \\
\hline 362.94 & 365.26 & 396.46 & 400.12 & 412.70 & 425.38 \\
\hline 432.05 & 440.28 & 450.85 & 461.79 & 464.15 & 473.27 \\
\hline 476.41 & 483.71 & 498.41 & 499.34 & 507.99 & 514.64 \\
\hline 530.29 & 538.72 & 545.03 & 560.33 & 577.57 & 586.54 \\
\hline 591.12 & 606.74 & 610.37 & 622.54 & 639.54 & 647.70 \\
\hline 666.05 & 682.44 & 684.81 & 711.83 & 721.58 & 727.84 \\
\hline 739.62 & 750.27 & 751.09 & 753.85 & 784.69 & 800.09 \\
\hline 800.40 & 812.72 & 865.73 & 870.72 & 882.59 & 887.24 \\
\hline 888.17 & 895.21 & 896.52 & 898.69 & 914.41 & 919.66 \\
\hline 942.08 & 944.70 & 945.11 & 948.03 & 952.27 & 956.09 \\
\hline 960.29 & 967.40 & 971.24 & 980.25 & 984.83 & 993.01 \\
\hline 1000.15 & 1006.69 & 1007.60 & 1009.83 & 1013.78 & 1023.60 \\
\hline 1027.78 & 1034.42 & 1037.68 & 1039.83 & 1050.15 & 1054.25 \\
\hline 1056.46 & 1065.11 & 1066.75 & 1067.75 & 1072.01 & 1077.15 \\
\hline 1080.00 & 1085.24 & 1088.92 & 1092.73 & 1101.56 & 1111.63 \\
\hline
\end{tabular}




\begin{tabular}{llllll}
1122.85 & 1136.94 & 1138.63 & 1153.68 & 1164.47 & 1169.91 \\
1195.75 & 1202.17 & 1210.19 & 1211.78 & 1218.09 & 1225.25 \\
1229.00 & 1230.95 & 1240.73 & 1241.58 & 1247.72 & 1251.18 \\
1255.13 & 1273.43 & 1277.68 & 1294.69 & 1298.78 & 1326.73 \\
1338.25 & 1338.69 & 1353.29 & 1358.52 & 1361.20 & 1365.13 \\
1368.58 & 1378.28 & 1378.80 & 1379.74 & 1380.54 & 1382.71 \\
1383.60 & 1388.10 & 1389.11 & 1391.67 & 1394.33 & 1398.30 \\
1399.70 & 1406.91 & 1409.64 & 1410.67 & 1411.73 & 1412.26 \\
1421.59 & 1422.09 & 1436.77 & 1440.89 & 1441.96 & 1443.81 \\
1445.29 & 1450.01 & 1450.37 & 1479.91 & 1481.01 & 1488.06 \\
1488.22 & 1494.98 & 1495.04 & 1498.98 & 1499.32 & 1504.81 \\
1505.66 & 1513.13 & 1514.59 & 1519.56 & 1520.08 & 1521.84 \\
1522.40 & 1526.53 & 1527.27 & 1531.21 & 1533.98 & 1537.46 \\
\hline 1537.98 & 1746.42 & 1797.46 & 1797.93 & 1820.11 & 1824.20 \\
2993.48 & 3007.08 & 3016.68 & 3017.83 & 3028.79 & 3033.59 \\
3034.35 & 3036.50 & 3039.17 & 3043.14 & 3048.79 & 3052.88 \\
3054.31 & 3055.03 & 3055.65 & 3063.33 & 3063.48 & 3064.39 \\
3067.12 & 3068.60 & 3070.60 & 3076.98 & 3082.25 & 3092.60 \\
3102.03 & 3105.60 & 3106.05 & 3107.25 & 3108.94 & 3113.15 \\
3118.91 & 3121.03 & 3131.15 & 3131.67 & 3131.89 & 3139.85 \\
3142.18 & 3151.51 & 3166.79 & 3175.54 & 3176.44 & 3179.25 \\
3179.88 & 3183.35 & 3184.16 & 3191.81 & 3559.85 & 3608.31
\end{tabular}

cinnafragrin A conformer 2

\begin{tabular}{|c|c|c|c|c|c|}
\hline 18.60 & 29.70 & 35.34 & 38.83 & 45.55 & 50.64 \\
\hline 55.13 & 61.45 & 67.37 & 69.15 & 74.96 & 82.11 \\
\hline 97.21 & 100.18 & 110.24 & 117.10 & 125.38 & 135.70 \\
\hline 141.82 & 154.51 & 156.97 & 164.49 & 180.63 & 188.27 \\
\hline 191.62 & 198.81 & 208.68 & 214.65 & 221.67 & 234.20 \\
\hline 243.41 & 251.57 & 261.97 & 268.42 & 281.06 & 289.81 \\
\hline 298.40 & 303.01 & 308.69 & 310.16 & 312.14 & 315.80 \\
\hline 318.88 & 332.34 & 334.38 & 343.50 & 353.19 & 356.41 \\
\hline 360.78 & 367.17 & 370.93 & 397.96 & 401.89 & 414.61 \\
\hline 428.28 & 438.44 & 446.66 & 463.39 & 464.70 & 469.20 \\
\hline 474.12 & 478.17 & 489.12 & 497.35 & 504.29 & 513.67 \\
\hline 520.18 & 528.12 & 542.04 & 557.97 & 572.14 & 575.24 \\
\hline
\end{tabular}




\begin{tabular}{|c|c|c|c|c|c|}
\hline 588.52 & 589.77 & 606.88 & 609.92 & 622.61 & 630.53 \\
\hline 646.19 & 652.83 & 677.59 & 683.23 & 714.88 & 727.69 \\
\hline 735.72 & 748.82 & 751.06 & 766.29 & 789.40 & 800.51 \\
\hline 802.95 & 813.69 & 857.63 & 867.45 & 879.94 & 886.73 \\
\hline 888.09 & 889.52 & 893.90 & 907.24 & 918.01 & 919.52 \\
\hline 937.34 & 941.44 & 945.09 & 946.32 & 952.75 & 953.34 \\
\hline 958.50 & 969.42 & 973.25 & 983.05 & 986.21 & 991.82 \\
\hline 997.75 & 1001.82 & 1007.91 & 1009.49 & 1010.17 & 1019.09 \\
\hline 1022.46 & 1028.54 & 1036.23 & 1037.41 & 1044.85 & 1048.29 \\
\hline 1059.71 & 1063.62 & 1066.74 & 1067.79 & 1071.92 & 1080.53 \\
\hline 1081.29 & 1086.81 & 1089.05 & 1092.86 & 1110.36 & 1117.13 \\
\hline 1130.36 & 1134.43 & 1137.68 & 1153.51 & 1157.10 & 1174.06 \\
\hline 1194.64 & 1202.76 & 1207.51 & 1209.93 & 1219.99 & 1222.05 \\
\hline 1226.43 & 1227.95 & 1241.75 & 1242.99 & 1244.62 & 1252.10 \\
\hline 1273.48 & 1274.97 & 1296.28 & 1296.46 & 1299.22 & 1322.18 \\
\hline 1335.56 & 1337.90 & 1344.92 & 1356.74 & 1360.34 & 1362.22 \\
\hline 1367.03 & 1373.28 & 1379.16 & 1379.90 & 1380.81 & 1383.99 \\
\hline 1385.05 & 1386.11 & 1386.58 & 1389.43 & 1392.95 & 1394.21 \\
\hline 1397.44 & 1400.31 & 1401.72 & 1409.12 & 1411.18 & 1411.82 \\
\hline 1413.34 & 1420.57 & 1422.66 & 1423.55 & 1442.96 & 1443.79 \\
\hline 1449.78 & 1450.28 & 1460.50 & 1480.68 & 1480.78 & 1488.58 \\
\hline 1489.02 & 1494.84 & 1495.02 & 1499.55 & 1499.58 & 1503.54 \\
\hline 1503.75 & 1513.67 & 1513.88 & 1519.23 & 1519.77 & 1522.32 \\
\hline 1522.58 & 1525.54 & 1525.83 & 1531.75 & 1531.97 & 1538.26 \\
\hline 1538.62 & 1755.13 & 1790.04 & 1811.64 & 1824.55 & 1825.89 \\
\hline 2976.26 & 2986.16 & 3014.69 & 3018.57 & 3019.23 & 3032.36 \\
\hline 3035.82 & 3036.32 & 3037.90 & 3039.14 & 3041.73 & 3046.64 \\
\hline 3050.99 & 3054.19 & 3054.28 & 3061.76 & 3063.19 & 3063.40 \\
\hline 3066.13 & 3067.82 & 3071.01 & 3077.72 & 3079.36 & 3099.85 \\
\hline 3102.20 & 3105.64 & 3106.01 & 3107.43 & 3108.93 & 3113.31 \\
\hline 3115.96 & 3131.07 & 3131.73 & 3134.55 & 3138.13 & 3142.04 \\
\hline 3143.77 & 3153.35 & 3174.23 & 3176.60 & 3179.78 & 3179.85 \\
\hline 2.57 & 3184.72 & 3189.11 & 3213.12 & 3565.96 & 3662.75 \\
\hline
\end{tabular}




\section{References}

1 Burton, L. P.; White, J. D. Syntheses of the Insect Antifeedant ( \pm )-Cinnamodial and the Drimane Sesquiterpenoids ( \pm )-Isodrimenin and $( \pm)$ Fragolide. J. Org. Chem. 1985, 50. 357364

2 Cortes, M.; Razmilic, I.; Lopez, J. Synthesis of (-)-Ugandensidial. J. Nat. Prod. 1990, 53, 1369-1371.

${ }^{3}$ In this report, the NMR spectra are referenced from the residual protium resonance, $\mathrm{CDCl}_{3}$ : $\delta 7.24$, and carbon resonance, $\mathrm{CDCl}_{3}: \delta 77.23$.

4 Spectrometer frequency was $400.12 \mathrm{MHz}$.

5 Harinantenaina, L.; Takaoka, S. Cinnafragrins A-C, Dimeric and Trimeric Drimane Sesquiterpenoids from Cinnamosma Fragrans, and Structure Revision of Capsicodendrin. $J$. Nat. Prod. 2006, 69, 1193-1197.

${ }^{6}$ Mahmoud, I. I.; Kinghorn, A. D.; Cordell, G. A.; Farnsworth, N. R. Potential Anticancer Agents. XVI. Isolation of Bicyclofarnesane Sesquiterpenoids from Capsicodendron Dinisii. J. Nat. Prod. 1980, 43, 365-371.

77 Spectrometer frequency was $100.62 \mathrm{MHz}$.

8 Karmahapatra, S.; Kientz, C.; Shetty, S.; Yalowich, J. C.; Rakotondraibe, L. H. Capsicodendrin from Cinnamosma Fragrans Exhibits Antiproliferative and Cytotoxic Activity in Human Leukemia Cells: Modulation by Glutathione. J. Nat. Prod. 2018, 81, 625-629.

9 Parr, R. G.; Yang, W. Density Functional Theory of Atoms and Molecules. Oxford University Press: New York, 1989

10 Bochevarov, A. D.; Harder, E.; Hughes, T. F.; Greenwood, J. R.; Braden, D. A.; Philipp, D. M.; Rinaldo, D.; Halls, M. D.; Zhang, J.; Friesner, R. Jaguar: a high-performance quantum chemistry software program with strengths in life and materials sciences. Int. J. Quantum Chem. 2013, 113, 2110-2142.

11 Slater, J. C. Quantum Theory of Molecules and Solids, Vol. 4: The Self-Consistent Field for Molecules and Solids. McGraw-Hill: New York, 1974.

12 Vosko, S. H.; Wilk, L.; Nusair, M. Accurate spin-dependent electron liquid correlation energies for local spin density calculations: a critical analysis. Can. J. Phys. 1980, 58, 12001211.

13 Becke, A. D. Density-functional exchange-energy approximation with correct asymptotic behavior. Phys. Rev. A 1988, 38, 3098-3100.

14 Becke, A. D. Density-functional thermochemistry. III. The role of exact exchange. J. Chem. Phys. 1993, 98, 5648-5652.

15 Lee, C.; Yang, W.; Parr, R. G. Development of the Colle-Salvetti correlation-energy formula into a functional of the electron density. Phys. Rev. B 1988, 37, 785. 
16 Grimme, S.; Antony, J.; Ehrlich, S.; Krieg, H. A consistent and accurate $a b$ initio parametrization of density functional dispersion correction (DFT-D) for the 94 elements $\mathrm{H}-\mathrm{Pu}$. J. Chem. Phys. 2010, 132, 154104.

17 Dunning, T. H., Jr. Gaussian basis sets for use in correlated molecular calculations. I. The atoms boron through neon and hydrogen. J. Phys. Chem. 1989, 90, 1007.

${ }^{18}$ Marten, B.; Kim, K.; Cortis, C.; Friesner, R. A.; Murphy, R. B.; Ringnalda, M. N.; Sitkoff, D.; Honig, B. New model for calculation of solvation free energies: correction of selfconsistent reaction field continuum dielectric theory for short-range hydrogen-bonding effects. J. Phys. Chem. 1996, 100, 11775-11788.

19 Edinger, S. R.; Cortis, C.; Shenkin, P. S.; Friesner, R. A. Solvation free energies of peptides: Comparison of approximate continuum solvation models with accurate solution of the Poisson- Boltzmann equation. J. Phys. Chem. B 1997, 101, 1190-1197.

${ }^{20}$ Friedrichs, M.; Zhou, R.; Edinger, S. R.; Friesner, R. A. Poisson- Boltzmann analytical gradients for molecular modeling calculations. J. Phys. Chem. B 1999, 103, 3057-3061.

${ }^{21}$ Rashin, A. A.; Honig, B. Reevaluation of the Born model of ion hydration. J. Phys. Chem. 1985, 89, 5588-5593.

22 Harinantenaina, L.; Takaoka, S. Cinnafragrins A-C, Dimeric and Trimeric Drimane Sesquiterpenoids from Cinnamosma fragrans, and Structure Revision of Capsicodendrin. $J$. Nat. Prod. 2006, 69, 1193-1197. 
7. Copies of ${ }^{1} \mathrm{H}$ and ${ }^{13} \mathrm{C}$ NMR Spectra of Newly Synthesized Compounds 


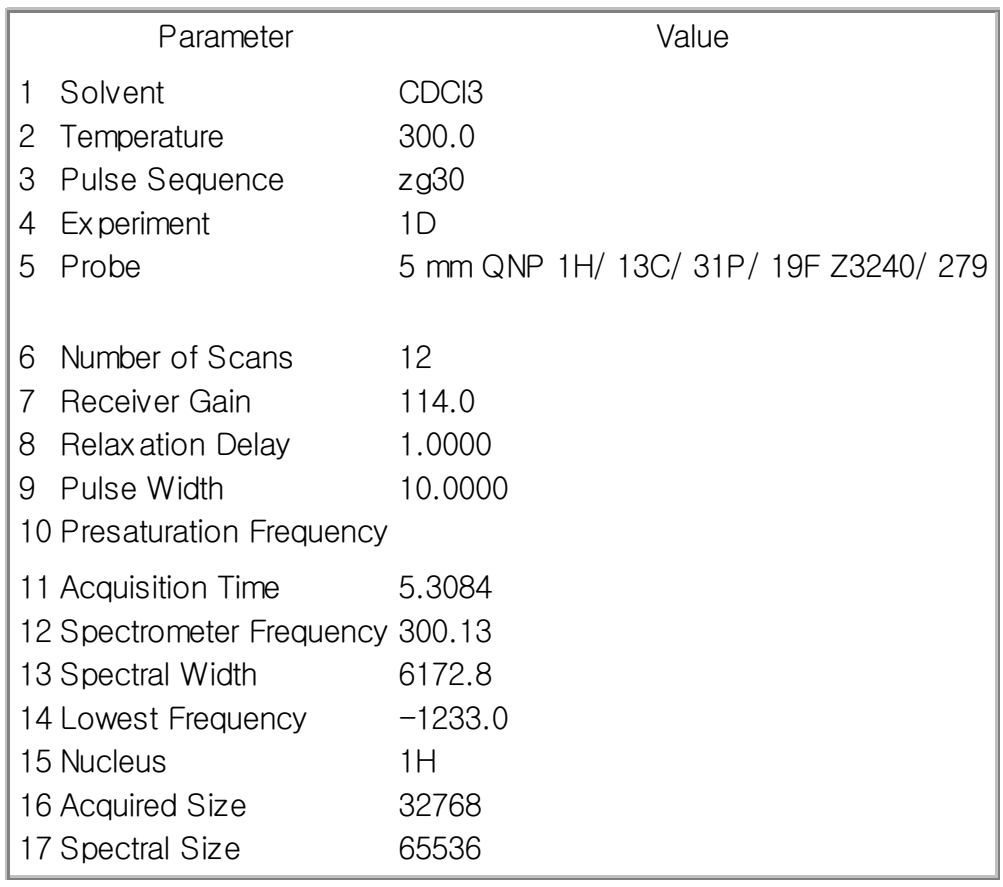

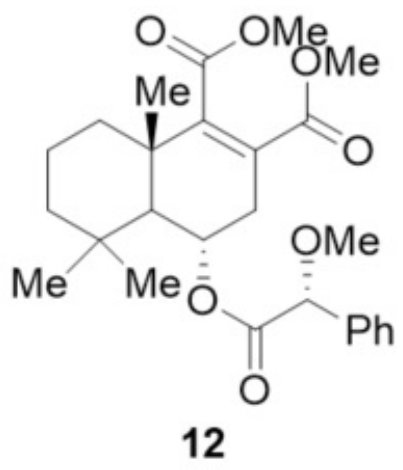


Total Synthesis of Cinnamodial-Based Dimer (-)-Capsicodendrin Jang, Y.; Han. S.

\begin{tabular}{|c|c|c|}
\hline 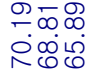 & \begin{tabular}{l} 
ç \\
\multirow{2}{*}{}
\end{tabular} & $\begin{array}{l}\infty \\
\infty \\
\text { in }\end{array}$ \\
\hline $1 / 1$ & & | \\
\hline
\end{tabular}

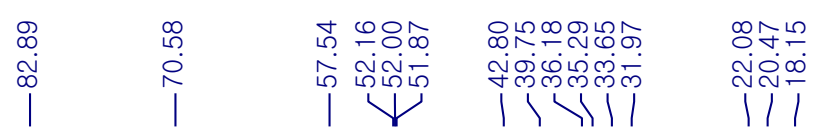

\begin{tabular}{|lll|}
\hline \multicolumn{1}{|c|}{ Parameter } & & Value \\
1 & Solvent & CDCl3 \\
2 & Temperature & 298.0 \\
3 & Pulse Sequence & zgpg30 \\
4 Experiment & 10 \\
5 Probe & Z116098_0402 (PA BBO 400S1 BBF-H-D-05 Z SP) \\
6 Number of Scans & 512 \\
7 Receiver Gain & 207.1 \\
8 Relaxation Delay & 2.0000 \\
9 Pulse Width & 10.0000 \\
10 Presaturation Frequency & \\
11 Acquisition Time & 1.3631 \\
12 Spectrometer Frequency & 100.62 \\
13 Spectral Width & 24038.5 \\
14 Lowest Frequency & -1943.5 \\
15 Nucleus & $13 \mathrm{C}$ \\
16 Acquired Size & 32768 \\
17 Spectral Size & 65536 \\
\hline
\end{tabular}

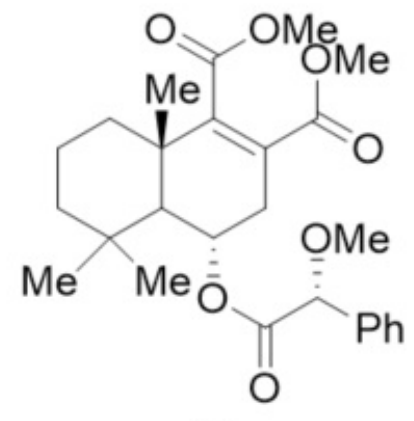

12

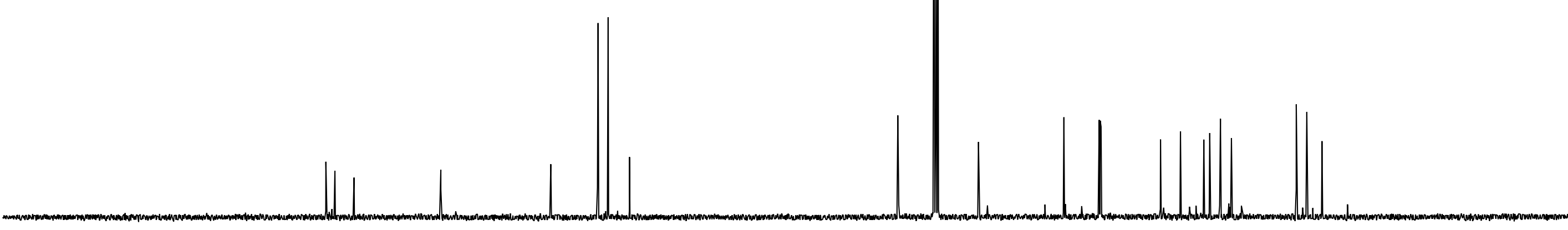

$210 \quad 200$ 
Total Synthesis of Cinnamodial-Based Dimer (-)-Capsicodendrin Jang, Y.; Han. S.*

\begin{tabular}{|lll|}
\hline \multicolumn{1}{|c|}{ Parameter } & & Value \\
1 & Solvent & CDCl3 \\
2 & Temperature & 298.0 \\
3 & Pulse Sequence & Zg30 \\
4 & Experiment & 10 \\
5 & Probe & Z116098_0402 (PA BBO 400S1 BBF-H-D-05 Z SP) \\
6 & Number of Scans & 16 \\
7 & Receiver Gain & 207.1 \\
8 & Relaxation Delay & 1.0000 \\
9 & Pulse Width & 9.6900 \\
10 Presaturation Frequency & \\
11 Acquisition Time & 4.0894 \\
12 Spectrometer Frequency & 400.12 \\
13 Spectral Width & 8012.8 \\
14 Lowest Frequency & -1553.3 \\
15 Nucleus & $1 \mathrm{H}$ \\
16 Acquired Size & 32768 \\
17 Spectral Size & 65536 \\
\hline
\end{tabular}

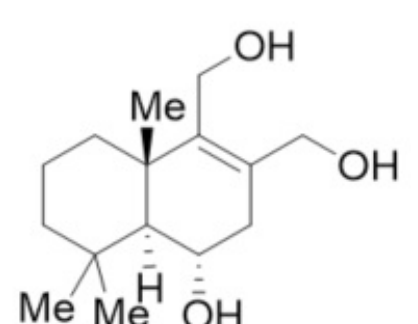

13

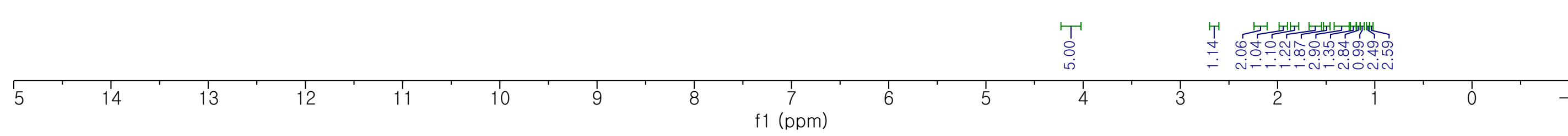




\begin{tabular}{|lll|}
\hline \multicolumn{1}{|c|}{ Parameter } & & Value \\
1 & Solvent & CDCl3 \\
2 & Temperature & 298.0 \\
3 & Pulse Sequence & zgpg30 \\
4 Experiment & 10 \\
5 Probe & Z116098_0402 (PA BBO 400S1 BBF-H-D-05 Z SP) \\
6 Number of Scans & 3072 \\
7 & Receiver Gain & 207.1 \\
8 & Relaxation Delay & 2.0000 \\
9 Pulse Width & 10.0000 \\
10 Presaturation Frequency \\
11 Acquisition Time & 1.3631 \\
12 Spectrometer Frequency & 100.62 \\
13 Spectral Width & 24038.5 \\
14 Lowest Frequency & -1937.4 \\
15 Nucleus & $13 \mathrm{C}$ \\
16 Acquired Size & 32768 \\
17 Spectral Size & 65536 \\
\hline
\end{tabular}

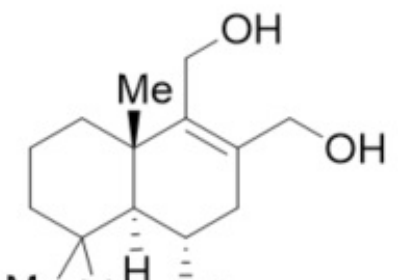

Me Me $\mathrm{H}^{\overline{\mathrm{O}}} \mathrm{H}$

13

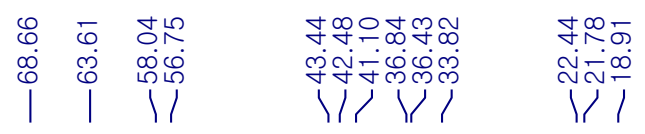

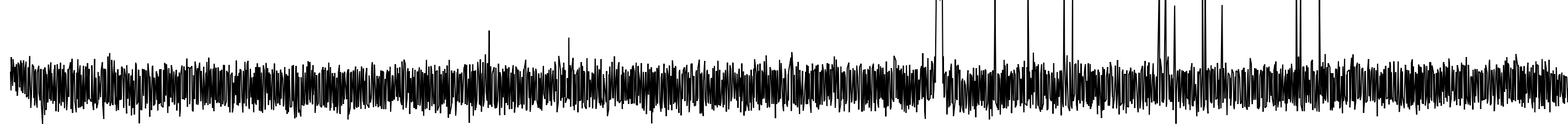


Total Synthesis of Cinnamodial-Based Dimer (-)-Capsicodendrin Jang, Y.; Han. S.*

\begin{tabular}{|lll|}
\hline \multicolumn{1}{|c|}{ Parameter } & & Value \\
1 & Solvent & CDCl3 \\
2 & Temperature & 298.0 \\
3 & Pulse Sequence & zg30 \\
4 Experiment & 10 \\
5 & Probe & Z116098_0402 (PA BBO 400S1 BBF-H-D-05 Z SP) \\
6 & Number of Scans & 16 \\
7 & Receiver Gain & 114.6 \\
8 & Relaxation Delay & 1.0000 \\
9 Pulse Width & 9.6900 \\
10 Presaturation Frequency & \\
11 Acquisition Time & 4.0894 \\
12 Spectrometer Frequency & 400.12 \\
13 Spectral Width & 8012.8 \\
14 Lowest Frequency & -1553.3 \\
15 Nucleus & $1 \mathrm{H}$ \\
16 Acquired Size & 32768 \\
17 Spectral Size & 65536 \\
\hline
\end{tabular}

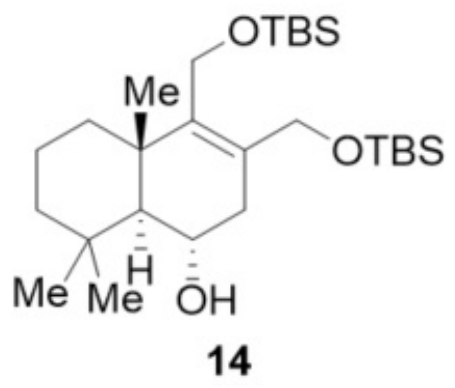

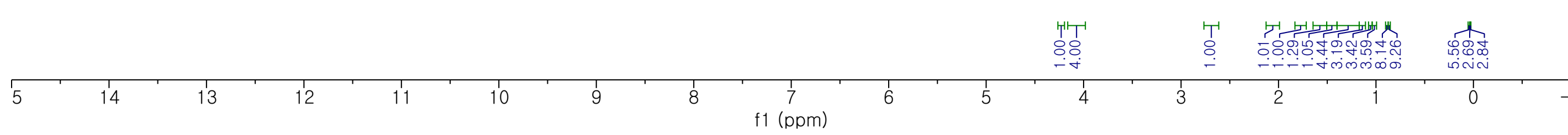




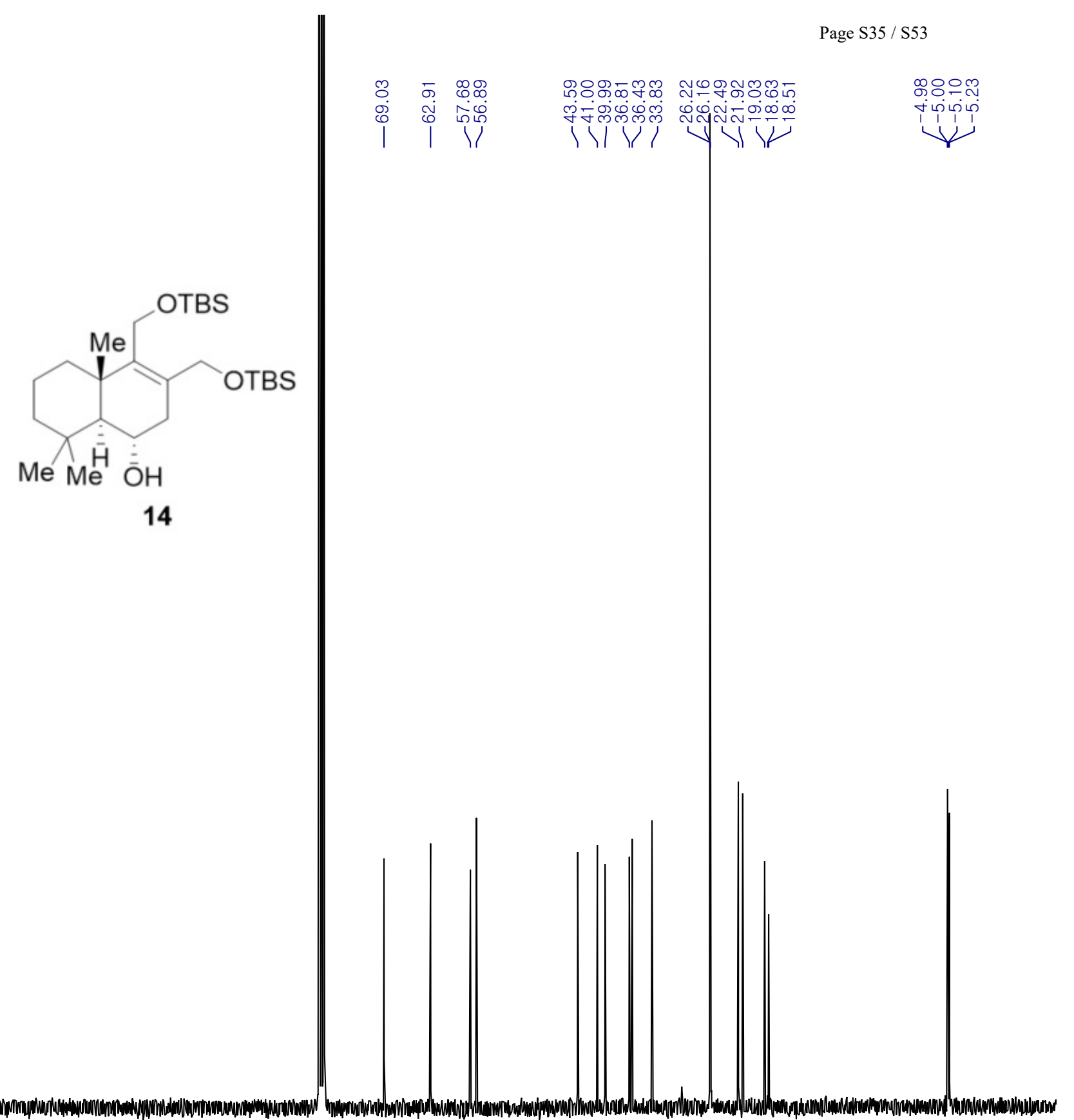




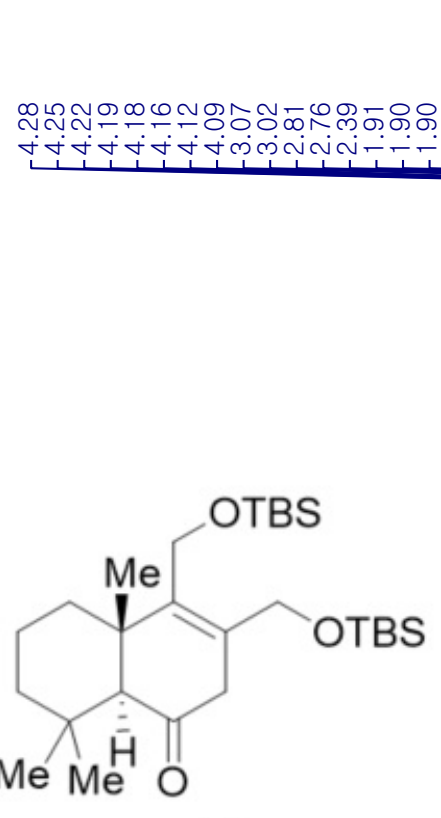

15

12 Spectrometer Frequency 400.12

13 Spectral Width $\quad 8012.8$

14 Lowest Frequency $\quad-1553.2$

15 Nucleus

$1 \mathrm{H}$

16 Acquired Size

32768

17 Spectral Size

65536

\section{Value}

298.0

g30

Z116098_0402 (PA BBO 400S1 BBF-H-D-05 Z SP)

16

30.7

1.0000

9.6900

894

553.2

$\begin{array}{lll}1 & \text { Solvent } & \mathrm{CDCl} 3 \\ 2 & \text { Temperature } & 298.0 \\ 3 & \text { Pulse Sequence } & \mathrm{zg} 30 \\ 4 & \text { Experiment } & 1 \mathrm{D} \\ 5 & \text { Probe } & \mathrm{Z} 116098 \\ 6 & \text { Number of Scans } & 16 \\ 7 & \text { Receiver Gain } & 130.7 \\ 8 & \text { Relaxation Delay } & 1.0000 \\ 9 \text { Pulse Width } & 9.6900 \\ 10 \text { Presaturation Frequency } \\ 11 \text { Acquisition Time } & 4.0894 \\ 12 \text { Spectrometer Frequency } & 400.12 \\ 13 \text { Spectral Width } & 8012.8 \\ 14 \text { Lowest Frequency } & -1553.2 \\ 15 \text { Nucleus } & 1 \mathrm{H} \\ 16 \text { Acquired Size } & 32768 \\ 17 \text { Spectral Size } & 65536\end{array}$

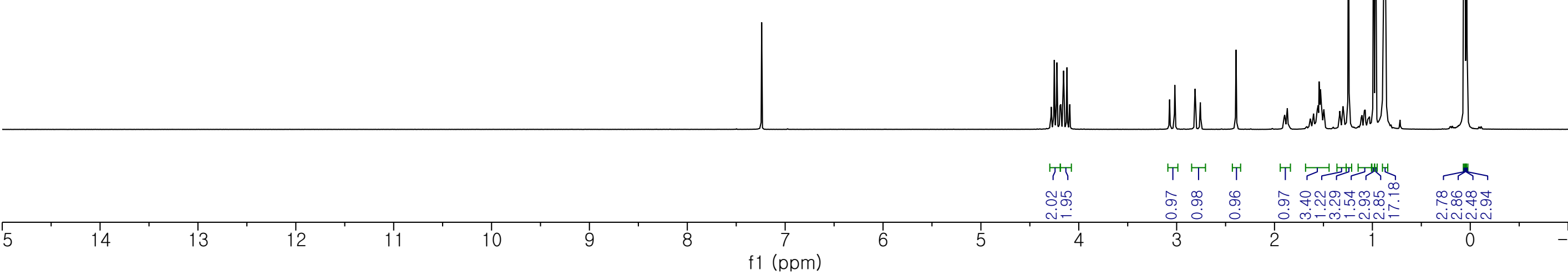


Total Synthesis of Cinnamodial-Based Dimer (-)-Capsicodendrin Jang, Y.; Han. S.*

\begin{tabular}{|lll|}
\hline \multicolumn{1}{|c|}{ Parameter } & & Value \\
1 & Solvent & CDCl3 \\
2 & Temperature & 298.0 \\
3 & Pulse Sequence & zgpg30 \\
4 & Experiment & 10 \\
5 & Probe & Z116098_0402 (PA BBO 400S1 BBF-H-D-05 Z SP) \\
6 & Number of Scans & 1024 \\
7 & Receiver Gain & 207.1 \\
8 & Relaxation Delay & 2.0000 \\
9 Pulse Width & 10.0000 \\
10 Presaturation Frequency & \\
11 Acquisition Time & 1.3631 \\
12 Spectrometer Frequency & 100.62 \\
13 Spectral Width & 24038.5 \\
14 Lowest Frequency & -1937.3 \\
15 Nucleus & $13 \mathrm{C}$ \\
16 Acquired Size & 32768 \\
17 Spectral Size & 65536 \\
\hline
\end{tabular}

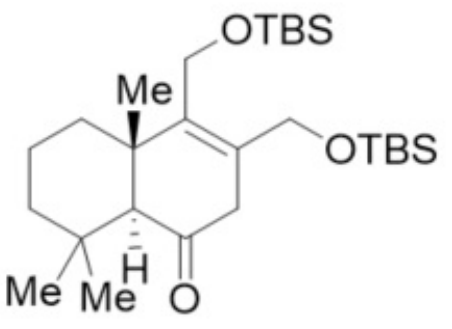

15
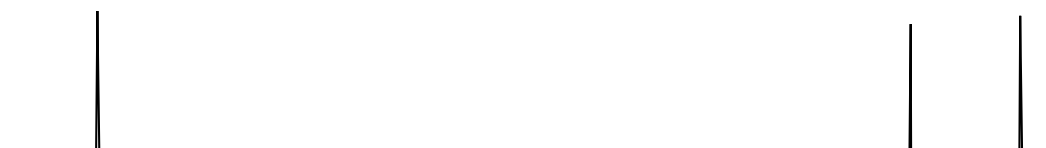


\begin{tabular}{|lll|}
\hline \multicolumn{1}{|c}{ Parameter } & & Value \\
1 & Solvent & CDCl3 \\
2 & Temperature & 298.0 \\
3 & Pulse Sequence & Zg30 \\
4 & Experiment & 10 \\
5 & Probe & Z116098_0402 (PA BBO 400S1 BBF-H-D-05 Z SP) \\
6 & Number of Scans & 16 \\
7 & Receiver Gain & 147.0 \\
8 & Relaxation Delay & 1.0000 \\
9 & Pulse Width & 9.6900 \\
10 & Presaturation Frequency & \\
11 Acquisition Time & 4.0894 \\
12 Spectrometer Frequency & 400.12 \\
13 Spectral Width & 8012.8 \\
14 Lowest Frequency & -1553.3 \\
15 Nucleus & $1 \mathrm{H}$ \\
16 Acquired Size & 32768 \\
17 Spectral Size & 65536 \\
\hline
\end{tabular}
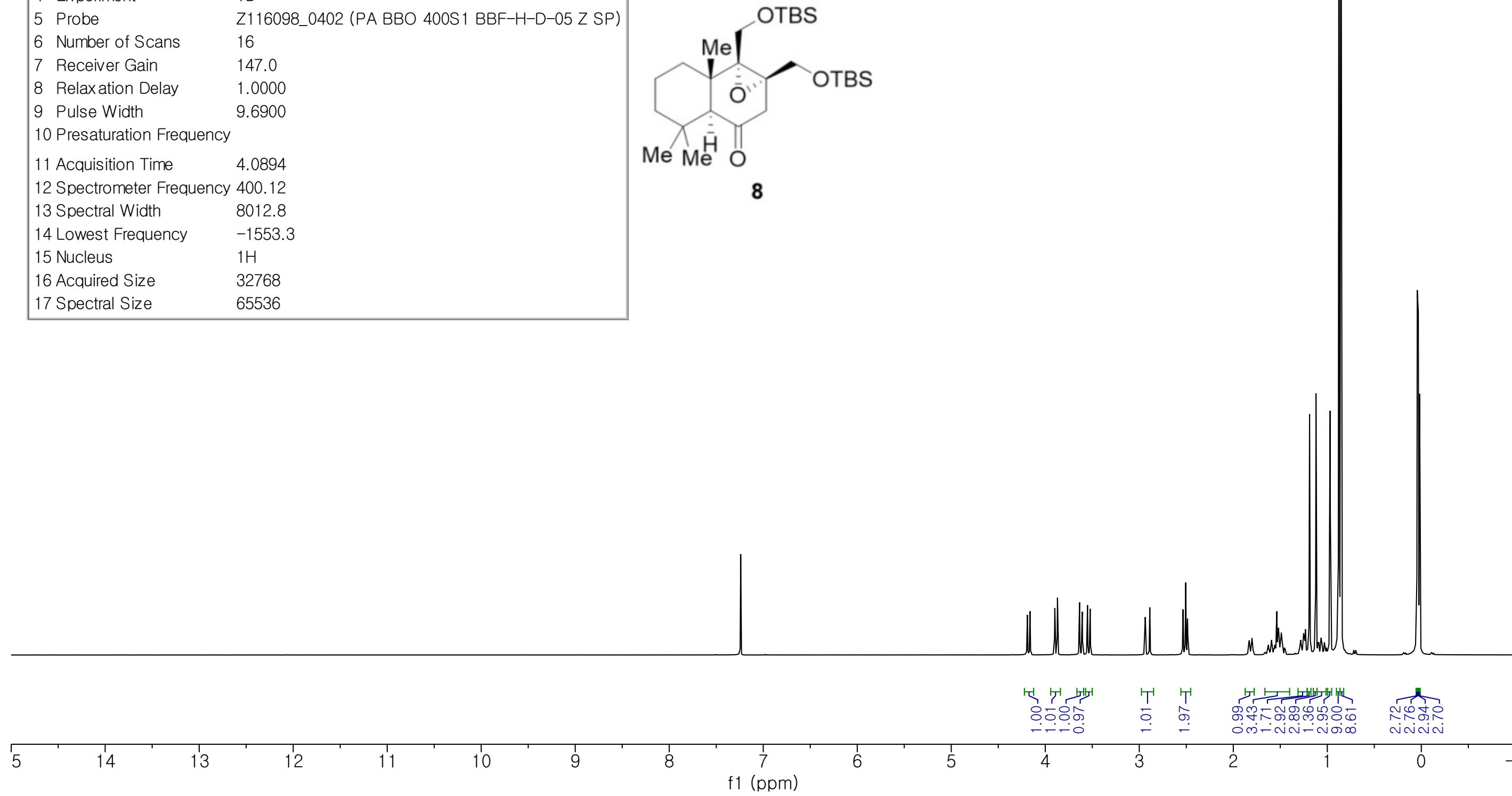
Total Synthesis of Cinnamodial-Based Dimer (-)-Capsicodendrin Jang, Y.; Han. S.*

\begin{tabular}{|lll|}
\hline \multicolumn{1}{|c|}{ Parameter } & & Value \\
1 & Solvent & CDCl3 \\
2 & Temperature & 298.0 \\
3 & Pulse Sequence & zgpg30 \\
4 & Experiment & 10 \\
5 & Probe & Z116098_0402 (PA BBO 400S1 BBF-H-D-05 Z SP) \\
6 & Number of Scans & 1024 \\
7 & Receiver Gain & 207.1 \\
8 & Relaxation Delay & 2.0000 \\
9 & Pulse Width & 10.0000 \\
10 Presaturation Frequency & \\
11 Acquisition Time & 1.3631 \\
12 Spectrometer Frequency & 100.62 \\
13 Spectral Width & 24038.5 \\
14 Lowest Frequency & -1937.3 \\
15 Nucleus & $13 \mathrm{C}$ \\
16 Acquired Size & 32768 \\
17 Spectral Size & 65536 \\
\hline
\end{tabular}

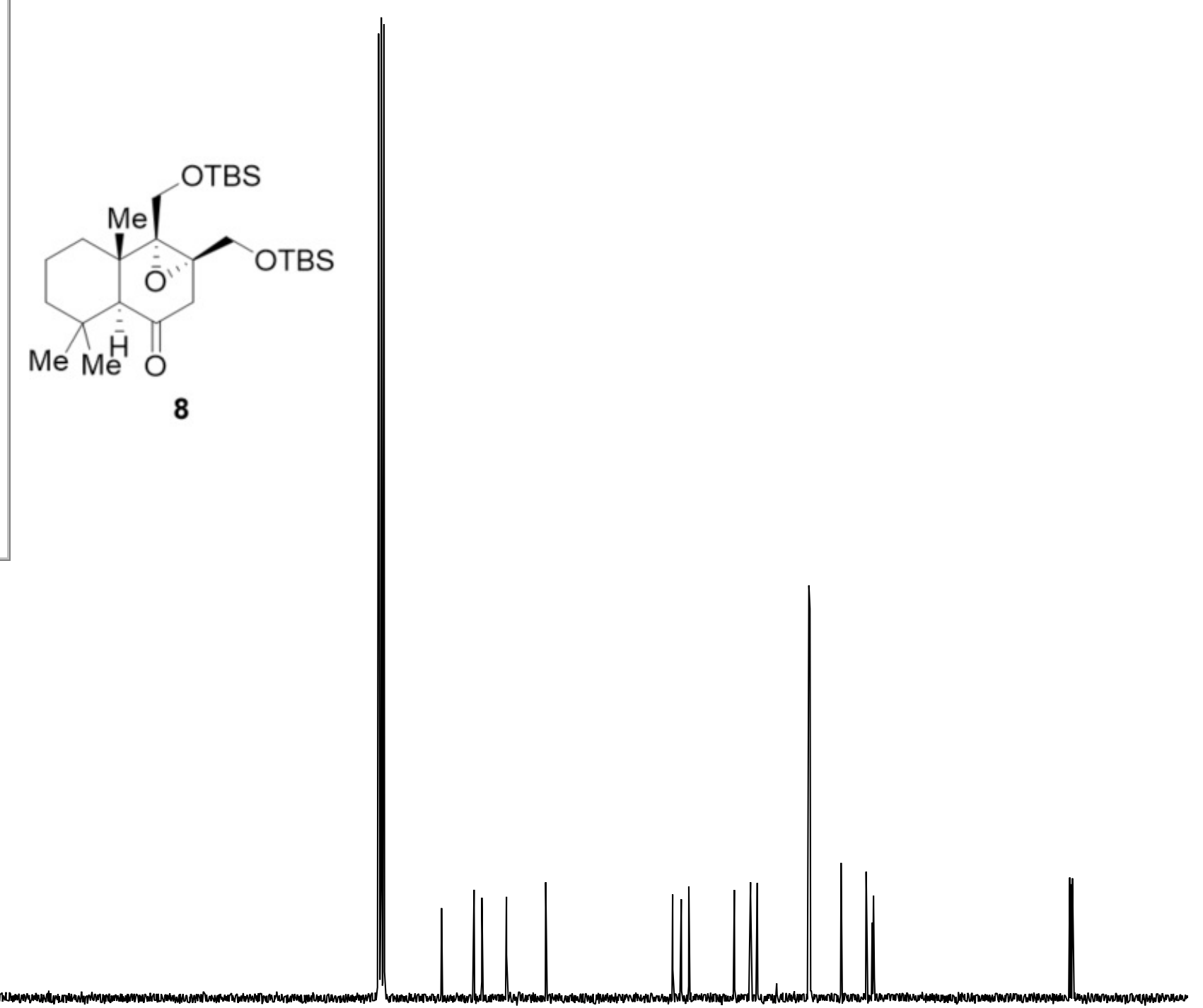




\begin{tabular}{|c|c|}
\hline Parameter & Value \\
\hline 1 Solvent & $\mathrm{CDCl} 3$ \\
\hline 2 Temperature & 298.0 \\
\hline 3 Pulse Sequence & zg30 \\
\hline 4 Experiment & 10 \\
\hline 5 Probe & Z116098_0402 (PA BBO 400S1 BBF-H-D-05 Z SP) \\
\hline 6 Number of Scans & 16 \\
\hline 7 Receiver Gain & 147.0 \\
\hline 8 Relaxation Delay & 1.0000 \\
\hline 9 Pulse Width & 9.6900 \\
\hline 10 Presaturation Frequency & \\
\hline 11 Acquisition Time & 4.0894 \\
\hline 12 Spectrometer Frequency & 400.12 \\
\hline 13 Spectral Width & 8012.8 \\
\hline 14 Lowest Frequency & -1553.3 \\
\hline 15 Nucleus & $1 \mathrm{H}$ \\
\hline 16 Acquired Size & 32768 \\
\hline 17 Spectral Size & 65536 \\
\hline
\end{tabular}
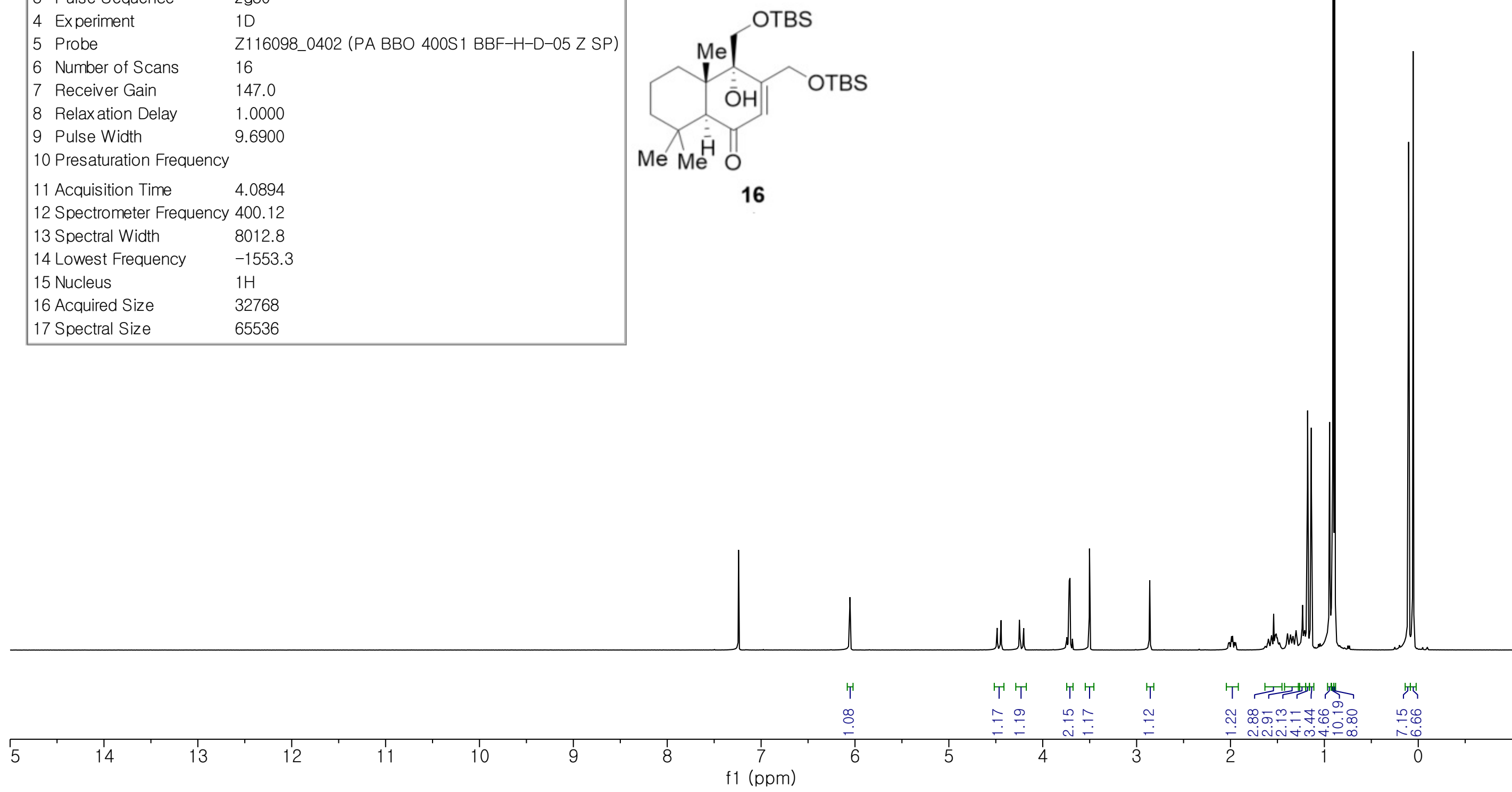

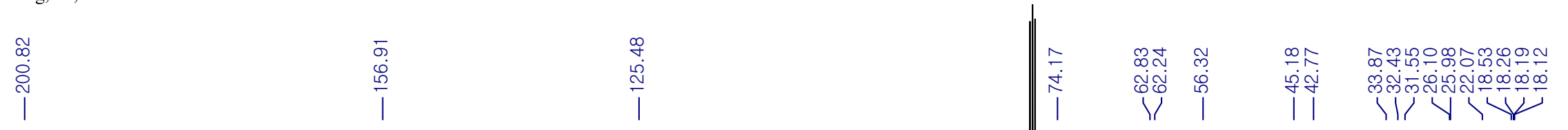

\begin{tabular}{|lll|}
\hline \multicolumn{2}{|c|}{ Parameter } & Value \\
1 & Solvent & $\mathrm{CDCl} 3$ \\
2 & Temperature & 298.0 \\
3 & Pulse Sequence & zgpg30 \\
4 Experiment & 10 \\
5 & Probe & Z116098_0402 (PA BBO 400S1 BBF-H-D-05 Z SP) \\
6 & Number of Scans & 1024 \\
7 & Receiver Gain & 207.1 \\
8 Relaxation Delay & 2.0000 \\
9 Pulse Width & 10.0000 \\
10 Presaturation Frequency & \\
11 Acquisition Time & 1.3631 \\
12 Spectrometer Frequency 100.62 \\
13 Spectral Width & 24038.5 \\
14 Lowest Frequency & -1937.5 \\
15 Nucleus & $13 \mathrm{C}$ \\
16 Acquired Size & 32768 \\
17 Spectral Size & 65536 \\
\hline
\end{tabular}

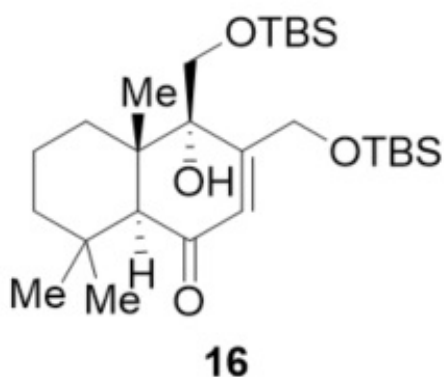




\begin{tabular}{|lll|}
\hline \multicolumn{1}{|c}{ Parameter } & & Value \\
1 & Solvent & CDCl3 \\
2 & Temperature & 298.0 \\
3 & Pulse Sequence & zg30 \\
4 Experiment & 10 \\
5 Probe & Z116098_0402 (PA BBO 400S1 BBF-H-D-05 Z SP) \\
6 Number of Scans & 16 \\
7 Receiver Gain & 130.7 \\
8 Relaxation Delay & 1.0000 \\
9 Pulse Width & 9.6900 \\
10 Presaturation Frequency & \\
11 Acquisition Time & 4.0894 \\
12 Spectrometer Frequency & 400.12 \\
13 Spectral Width & 8012.8 \\
14 Lowest Frequency & -1553.2 \\
15 Nucleus & $1 \mathrm{H}$ \\
16 Acquired Size & 32768 \\
17 Spectral Size & 65536 \\
\hline
\end{tabular}

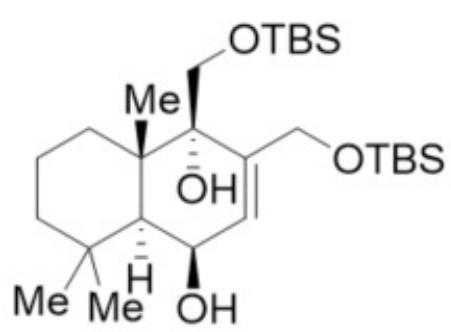

17

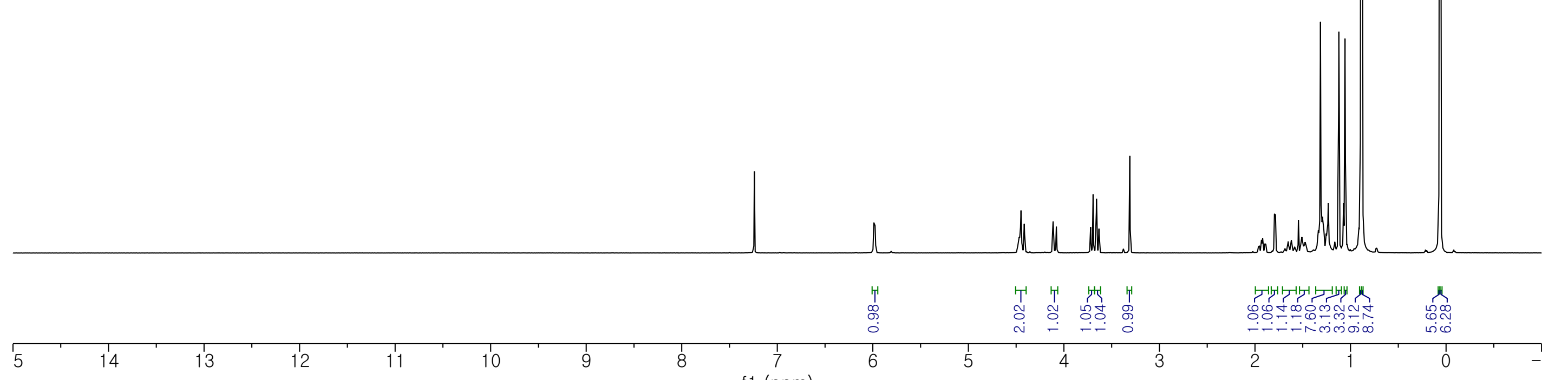

f1 (ppm) 


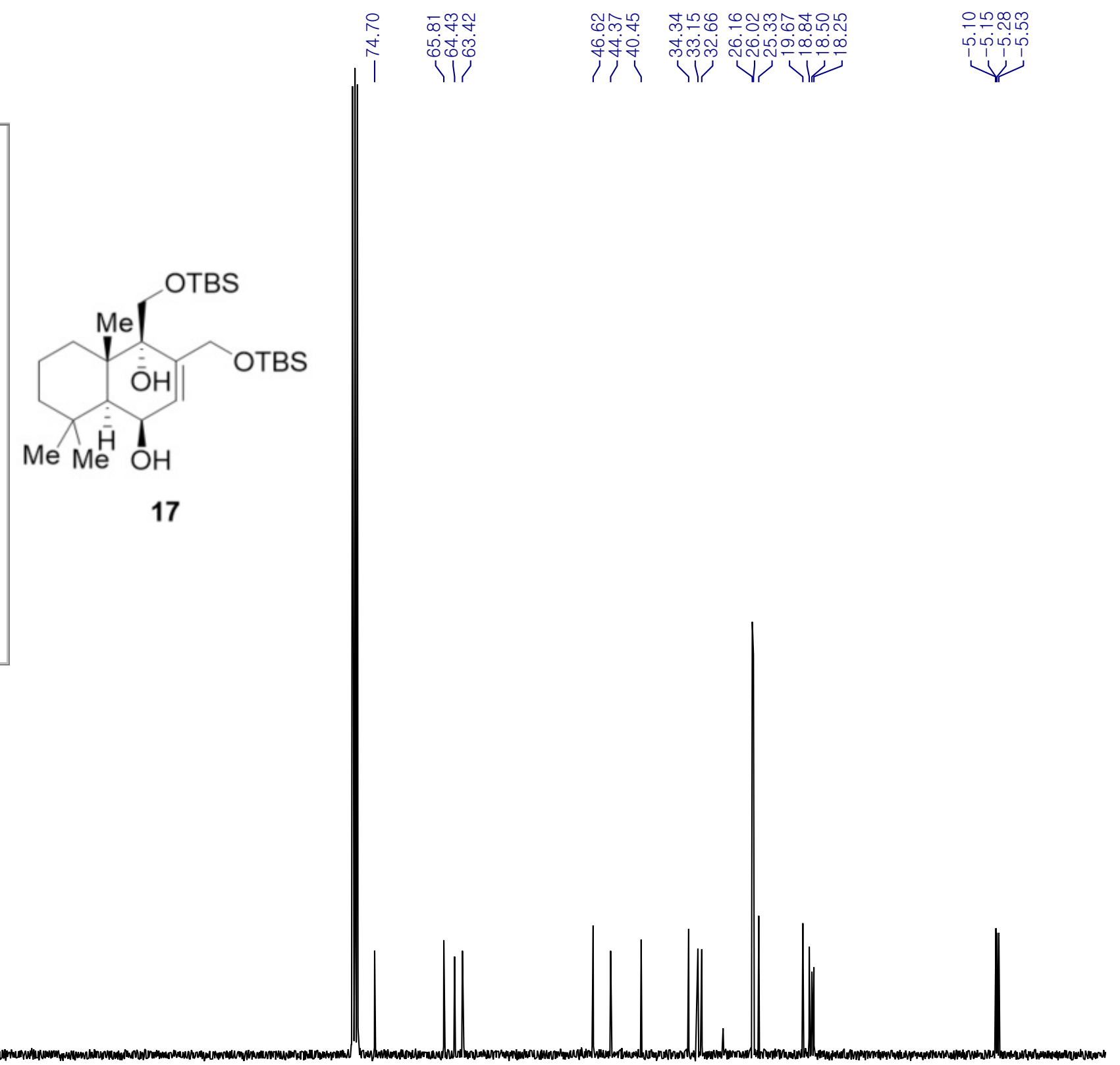

$210 \quad 200$

190




\begin{tabular}{|c|c|}
\hline Parameter & Value \\
\hline 1 Solvent & $\mathrm{CDCl} 3$ \\
\hline 2 Temperature & 298.0 \\
\hline 3 Pulse Sequence & zg30 \\
\hline 4 Experiment & 10 \\
\hline 5 Probe & Z116098_0402 (PA BBO 400S1 BBF-H-D-05 Z SP) \\
\hline 6 Number of Scans & 16 \\
\hline 7 Receiver Gain & 100.7 \\
\hline 8 Relaxation Delay & 1.0000 \\
\hline 9 Pulse Width & 9.6900 \\
\hline 10 Presaturation Frequency & \\
\hline 11 Acquisition Time & 4.0894 \\
\hline 12 Spectrometer Frequency & 400.12 \\
\hline 13 Spectral Width & 8012.8 \\
\hline 14 Lowest Frequency & -1553.2 \\
\hline 15 Nucleus & $1 \mathrm{H}$ \\
\hline 16 Acquired Size & 32768 \\
\hline 17 Spectral Size & 65536 \\
\hline
\end{tabular}

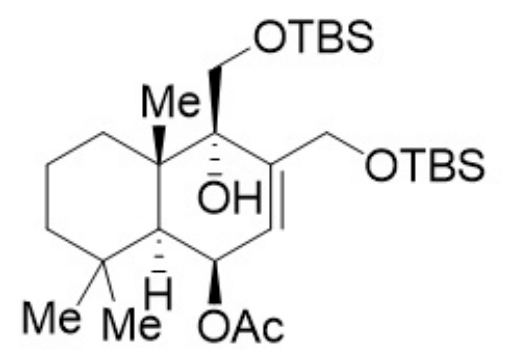

18

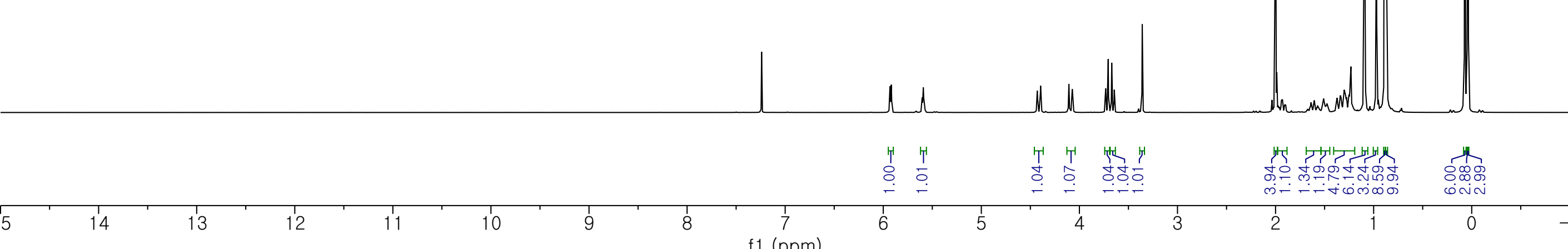

f1 (ppm) 


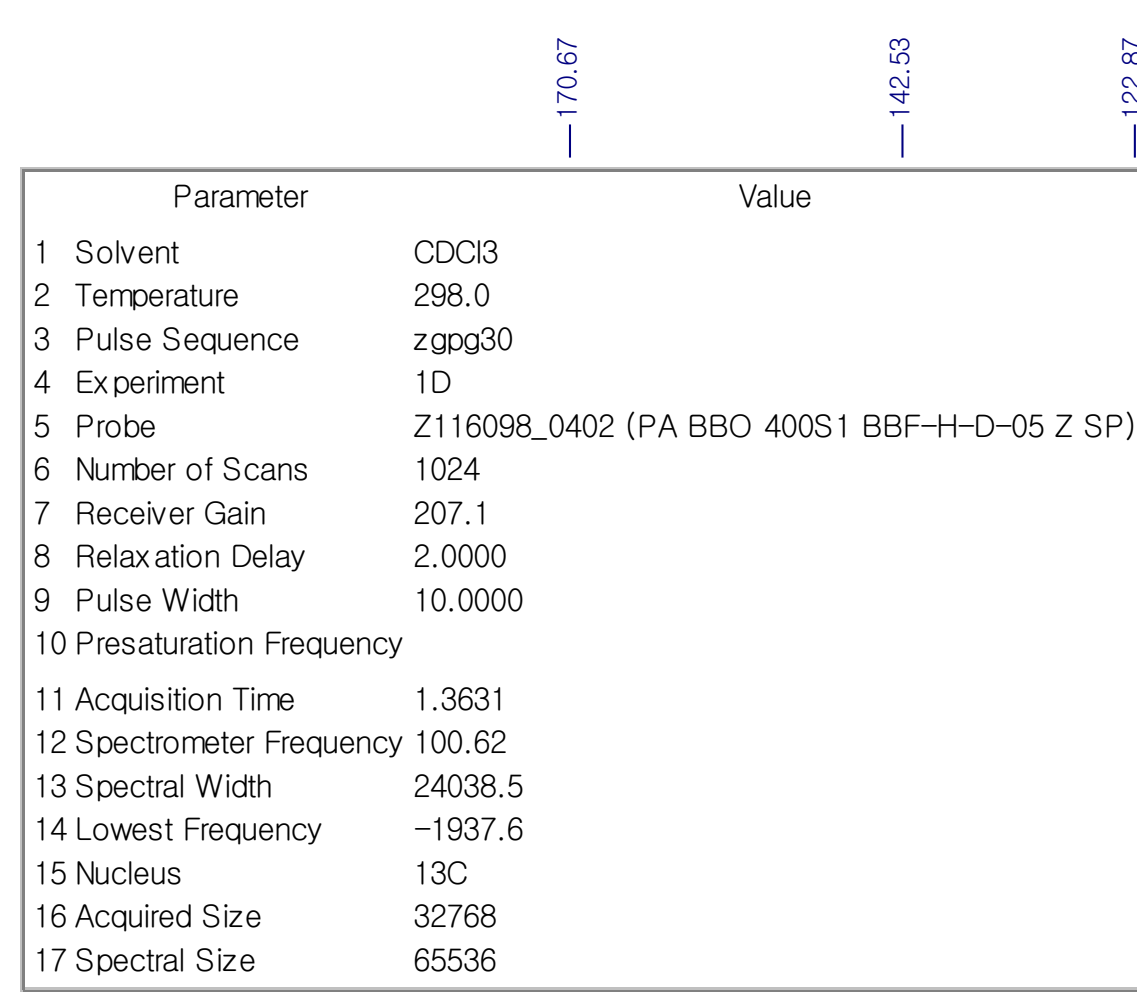

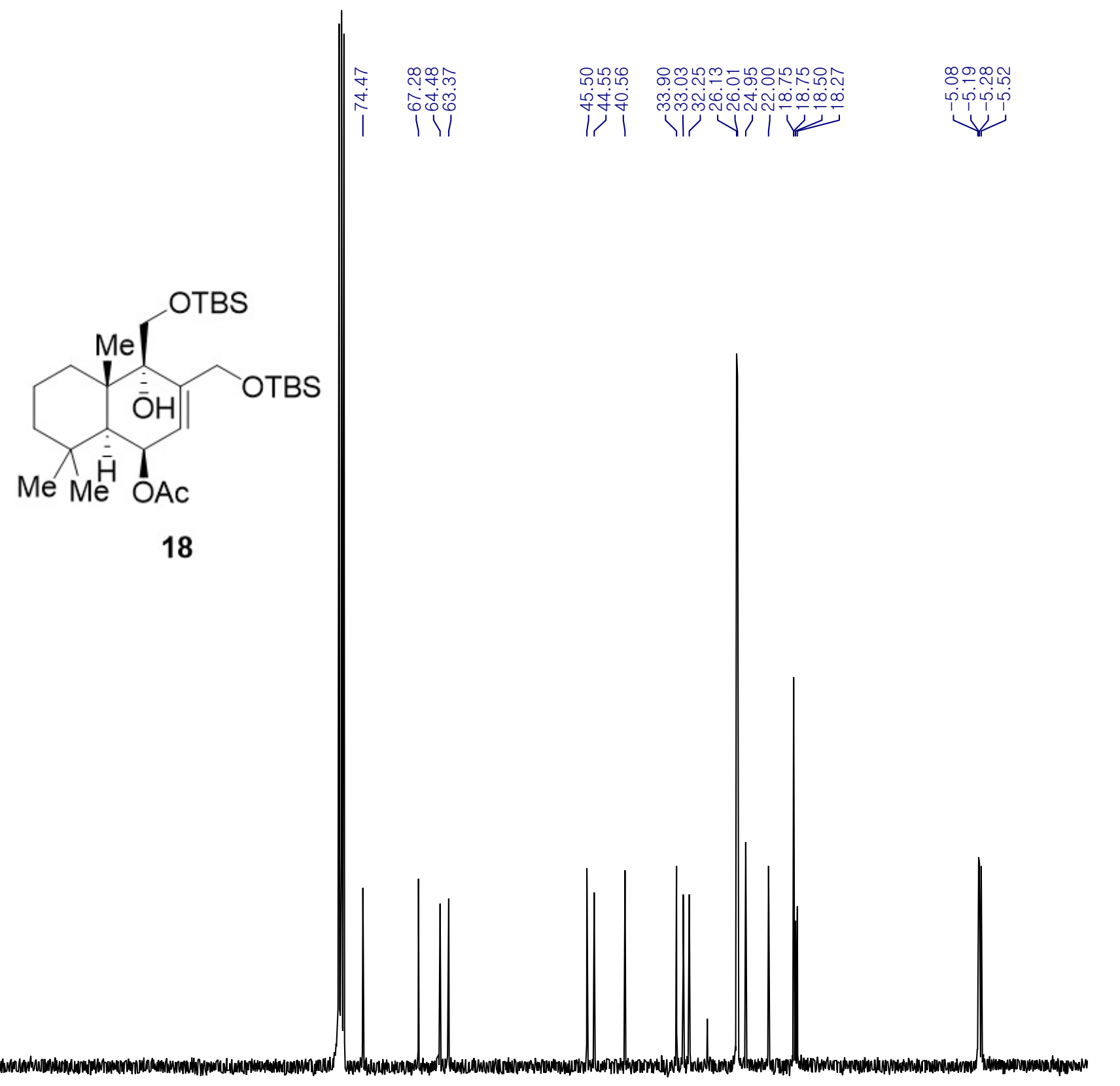




\begin{tabular}{|lll|}
\hline \multicolumn{1}{|c|}{ Parameter } & & Value \\
1 & Solvent & $\mathrm{CDCl} 3$ \\
2 & Temperature & 298.0 \\
3 & Pulse Sequence & $\mathrm{Zg} 30$ \\
4 & Experiment & $1 \mathrm{D}$ \\
5 & Probe & $\mathrm{Z} 116098$ 0402 (PA BBO 400S1 BBF-H-D-05 Z SP) \\
6 & Number of Scans & 16 \\
7 & Receiver Gain & 100.7 \\
8 & Relaxation Delay & 1.0000 \\
9 Pulse Width & 9.6900 \\
10 Presaturation Frequency & \\
11 Acquisition Time & 4.0894 \\
12 Spectrometer Frequency & 400.12 \\
13 Spectral Width & 8012.8 \\
14 Lowest Frequency & -1553.2 \\
15 Nucleus & $1 \mathrm{H}$ \\
16 Acquired Size & 32768 \\
17 Spectral Size & 65536 \\
\hline
\end{tabular}

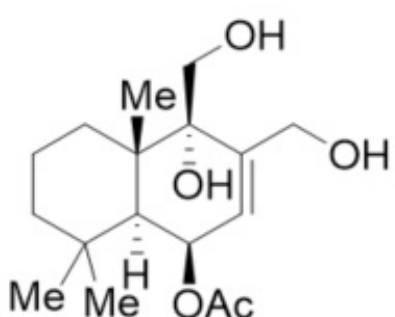

19

17 Spectral Size $\quad 65536$

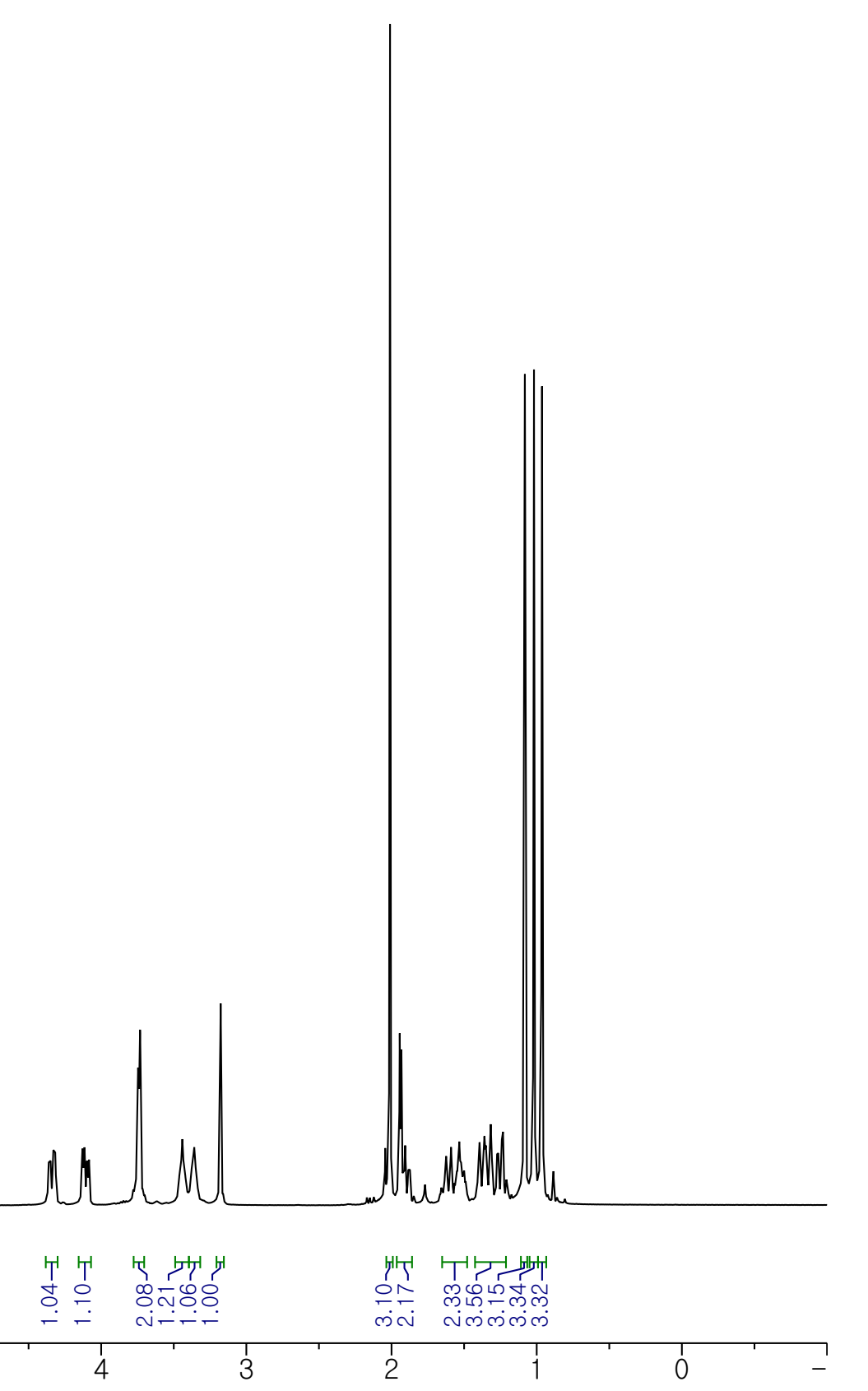


Total Synthesis of Cinnamodial-Based Dimer (-)-Capsicodendrin Jang, Y.; Han. S.*

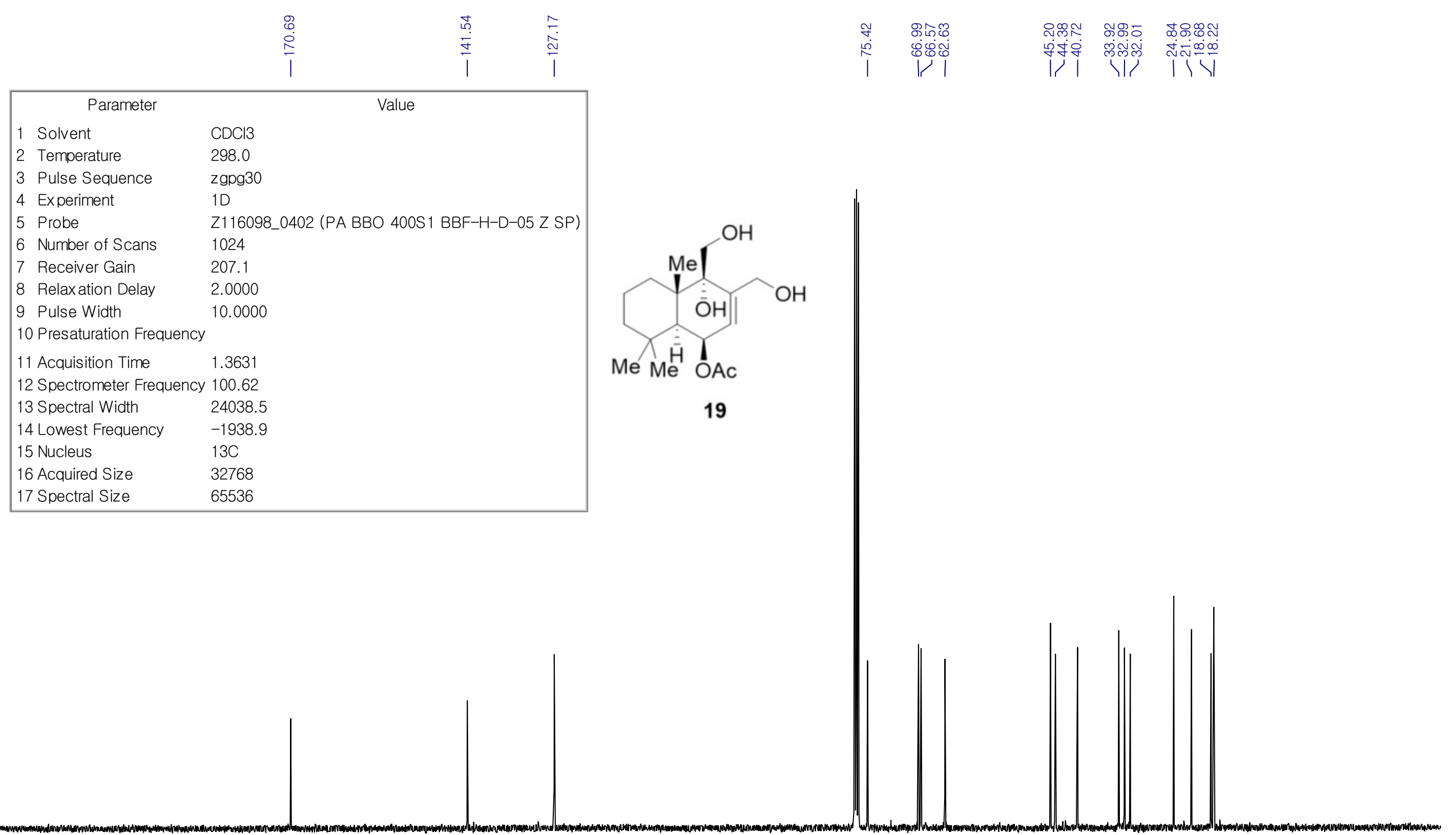


rom

\begin{tabular}{|lll|}
\hline \multicolumn{1}{|c|}{ Parameter } & \multicolumn{1}{c|}{ Value } \\
1 & Solvent & $\mathrm{CDCl} 3$ \\
2 & Temperature & 298.0 \\
3 & Pulse Sequence & $\mathrm{Zg} 30$ \\
4 & Experiment & $1 \mathrm{D}$ \\
5 & Probe & $\mathrm{Z} 116098 \_0402$ (PA BBO 400S1 BBF-H-D-05 Z SP) \\
6 & Number of Scans & 16 \\
7 & Receiver Gain & 31.9 \\
8 & Relaxation Delay & 1.0000 \\
9 & Pulse Width & 9.6900 \\
10 & Presaturation Frequency \\
11 & Acquisition Time & 4.0894 \\
12 & Spectrometer Frequency & 400.12 \\
13 & Spectral Width & 8012.8 \\
14 & Lowest Frequency & -1553.1 \\
15 & Nucleus & $1 \mathrm{H} .1$ \\
16 & Acquired Size & 32768 \\
17 & Spectral Size & 65536 \\
\hline
\end{tabular}

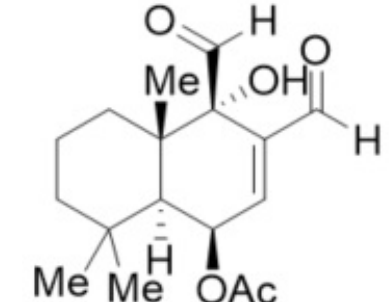

(-)-cinnamodial (1)

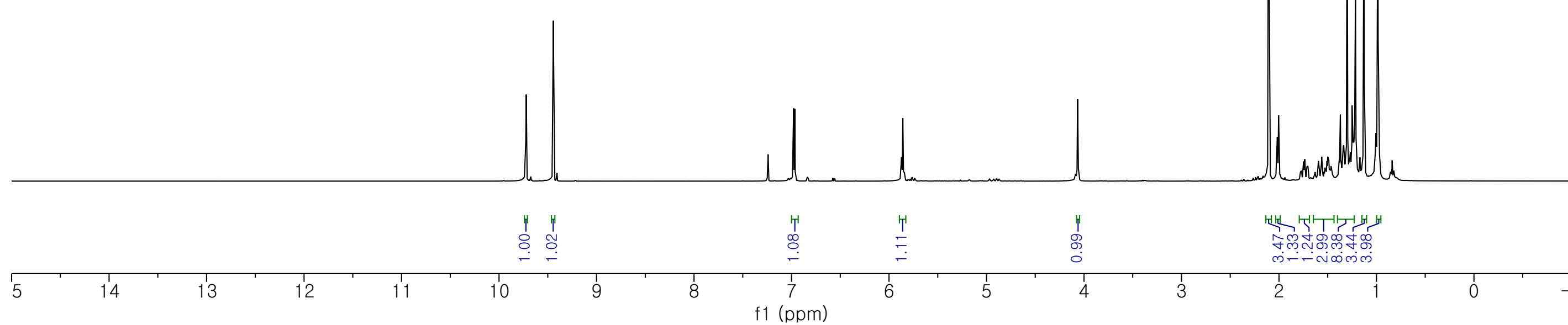




\begin{tabular}{|lll|}
\hline \multicolumn{1}{|c|}{ Parameter } & & Value \\
1 & Solvent & CDCl3 \\
2 & Temperature & 298.0 \\
3 & Pulse Sequence & zgpg30 \\
4 & Experiment & 10 \\
5 & Probe & Z116098_0402 (PA BBO 400S1 BBF-H-D-05 Z SP) \\
6 & Number of Scans & 1024 \\
7 & Receiver Gain & 207.1 \\
8 & Relaxation Delay & 2.0000 \\
9 & Pulse Width & 10.0000 \\
10 Presaturation Frequency & \\
11 Acquisition Time & 1.3631 \\
12 Spectrometer Frequency & 100.62 \\
13 Spectral Width & 24038.5 \\
14 Lowest Frequency & -1940.0 \\
15 Nucleus & $13 \mathrm{C}$ \\
16 Acquired Size & 32768 \\
17 Spectral Size & 65536 \\
\hline
\end{tabular}

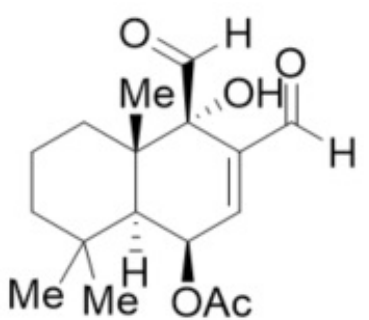

(-)-cinnamodial (1)

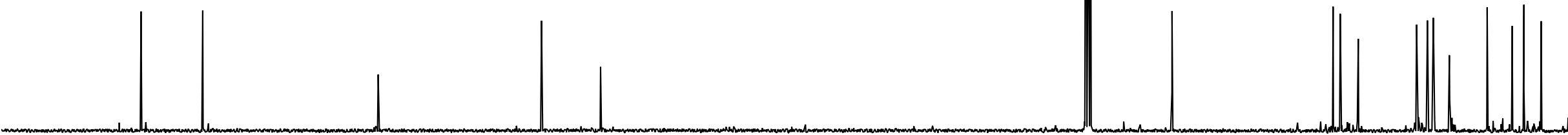




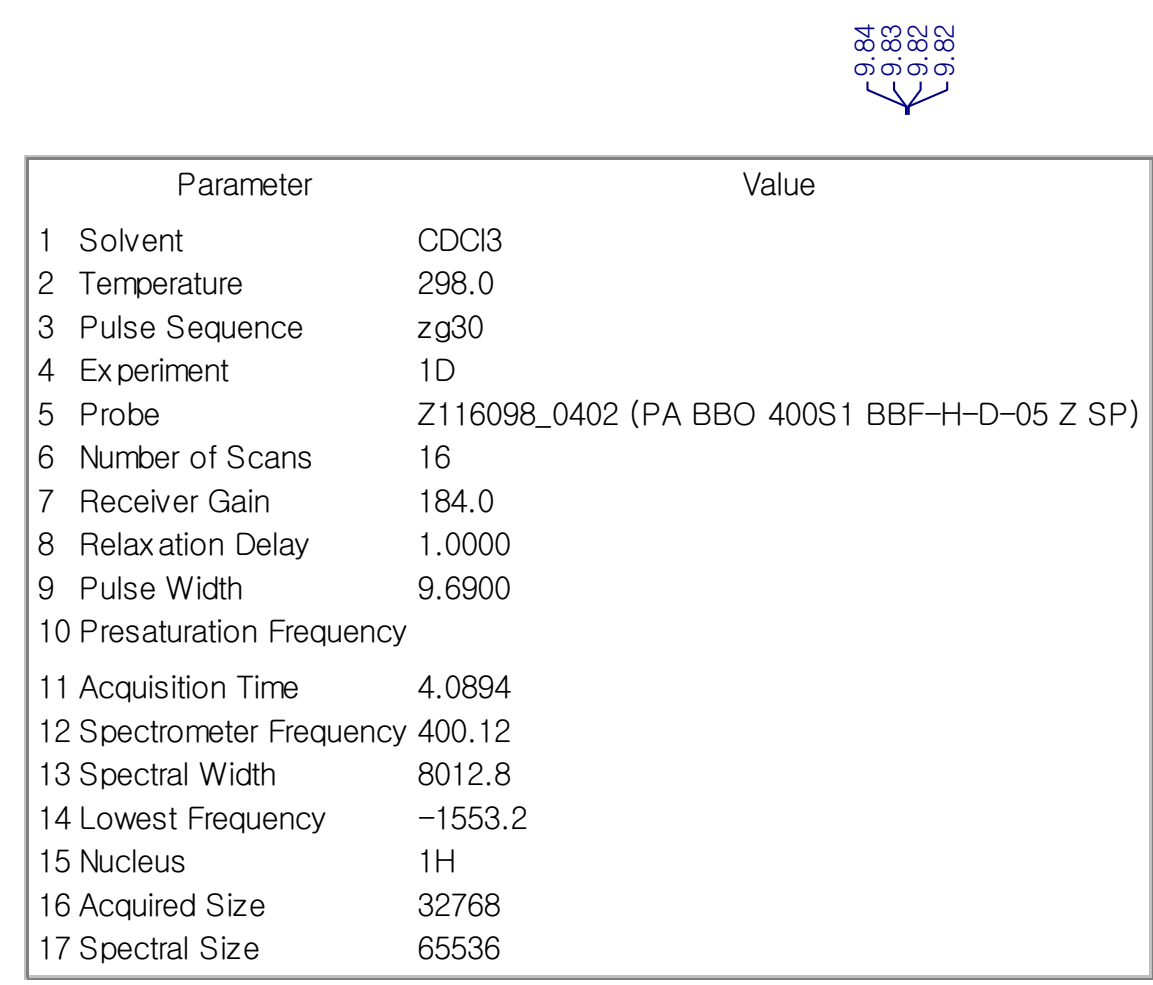

$\mathrm{CDCl} 3$

Pulse Sequence

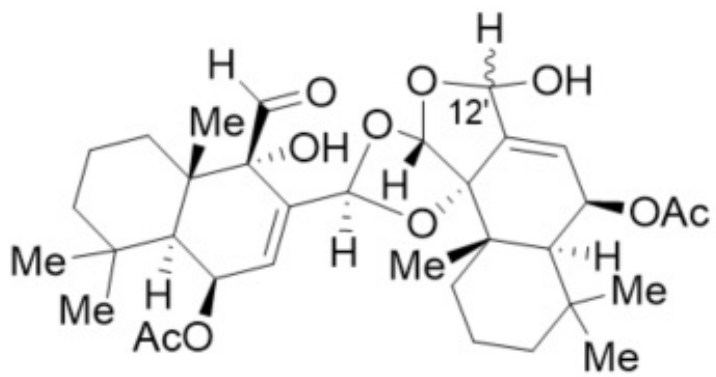

(-)-capsicodendrin (3)

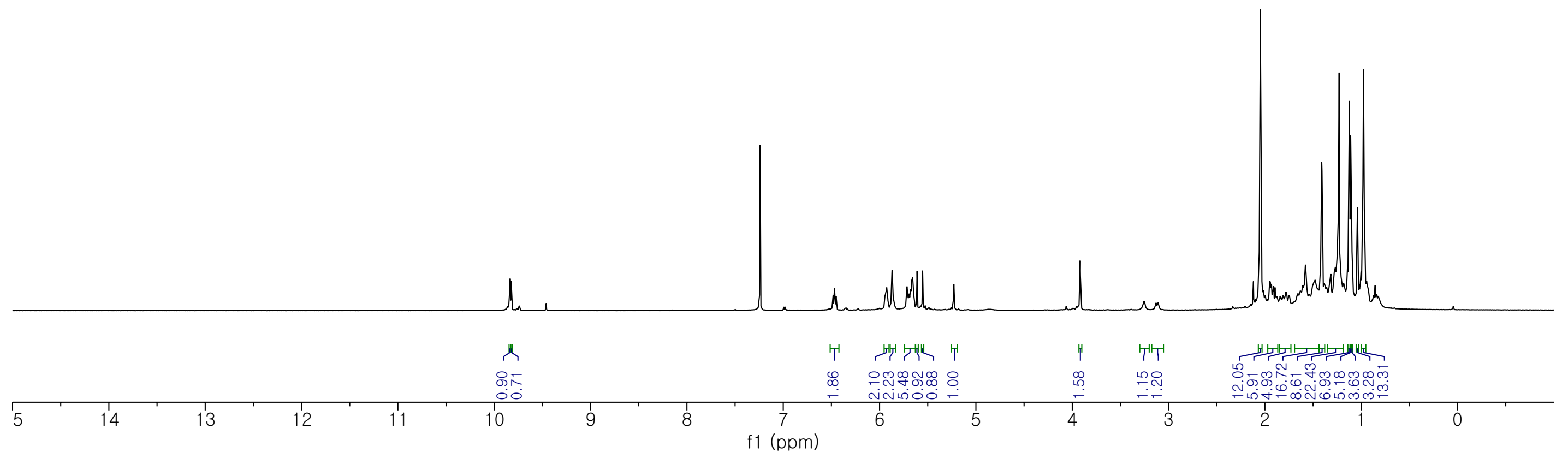




\section{Parameter}

1 Solvent

2 Temperature

3 Pulse Sequence

4 Experiment

5 Probe

6 Number of Scans

7 Receiver Gain

8 Relaxation Delay

9 Pulse Width

10 Presaturation Frequency

11 Acquisition Time

12 Spectrometer Frequency 100.62

13 Spectral Width $\quad 24038.5$

14 Lowest Frequency $\quad-1938.0$

15 Nucleus

16 Acquired Size

17 Spectral Size

$13 \mathrm{C}$

32768

65536

$\mathrm{CDCl} 3$

zgpg30 600

207.1

2.0000

.0000
Value

Z116098_0402 (PA BBO 400S1 BBF-H-D-05 Z SP) $111 \mathrm{~V}$

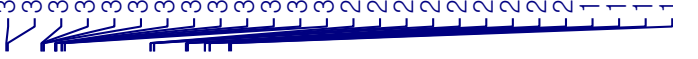

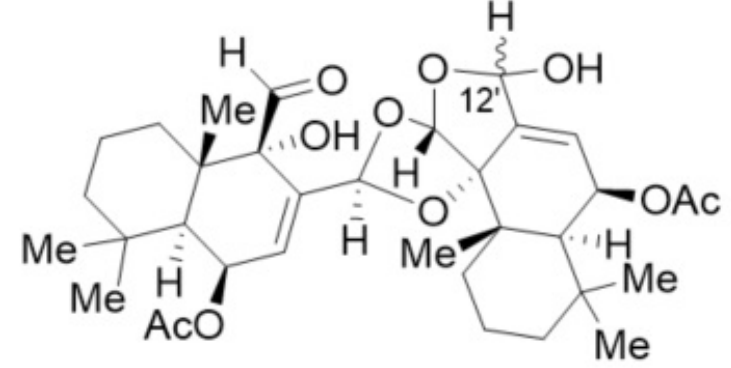

(-)-capsicodendrin (3)

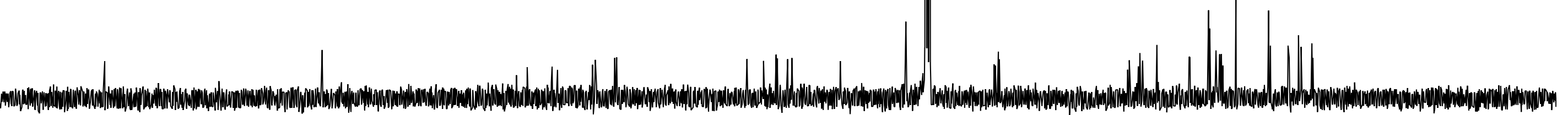




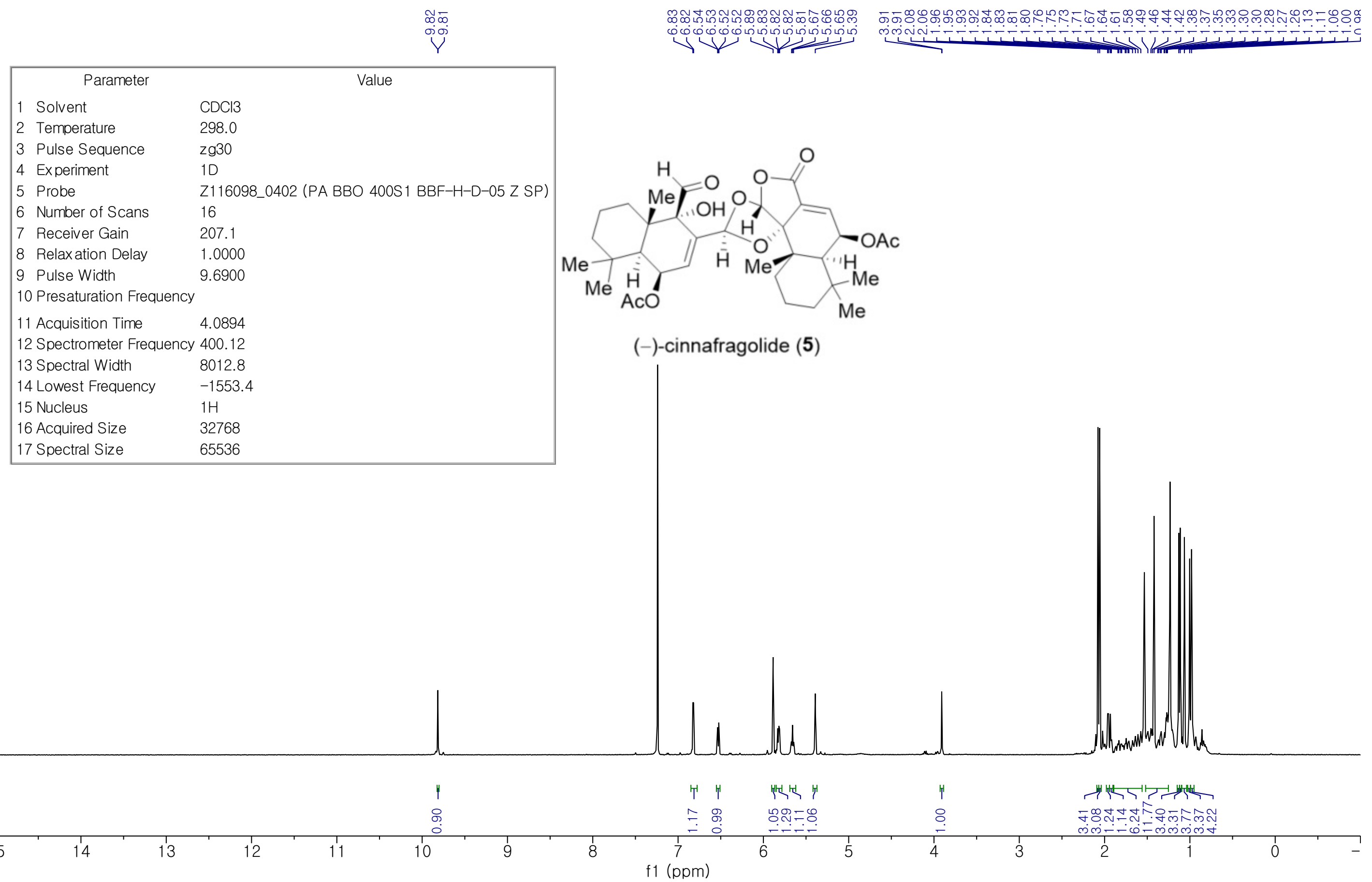



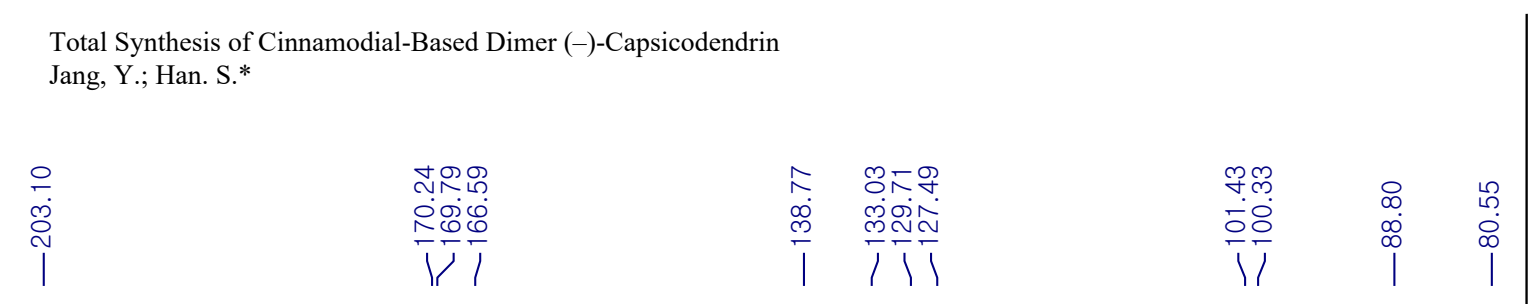

\section{$\mathrm{CDCl} 3$}

Temperature

298.0

Pulse Sequence

zgpg30

4 Experiment

5 Probe

6 Number of Scans

7 Receiver Gain

8 Relaxation Delay

1024

207.1

9 Pulse Width

2.0000

10 Presaturation Frequency

11 Acquisition Time $\quad 1.3631$

12 Spectrometer Frequency 100.62

13 Spectral Width $\quad 24038.5$

14 Lowest Frequency $\quad-1937.6$

15 Nucleus

$13 \mathrm{C}$

16 Acquired Size

17 Spectral Size

65536

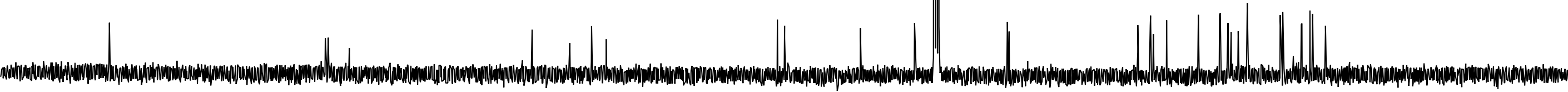

$210 \quad 200$
190
180
170
160
150
140
130
120
$110 \quad 100$

f1 (ppm)
$90 \quad 80$

70

60
40 